\title{
Local simulations of the magnetized Kelvin-Helmholtz instability in neutron-star mergers
}

\author{
M. Obergaulinger ${ }^{1}$, M. A. Aloy ${ }^{2}$, and E. Müller ${ }^{1}$ \\ 1 Max-Planck-Institut für Astrophysik, Garching bei München, Germany \\ e-mail: mobergau@MPA-Garching.MPG. de \\ 2 Departamento de Astronomía y Astrofísica, Universidad de Valencia, Spain \\ Received 1 October 2009 / Accepted 31 March 2010
}

\section{ABSTRACT}

\begin{abstract}
Context. Global magnetohydrodynamic simulations show the growth of Kelvin-Helmholtz instabilities at the contact surface of two merging neutron stars. That region has been identified as the site of efficient amplification of magnetic fields. However, these global simulations, due to numerical limitations, were unable to determine the saturation level of the field strength, and thus the possible back-reaction of the magnetic field onto the flow.

Aims. We investigate the amplification of initially weak magnetic fields in Kelvin-Helmholtz unstable shear flows, and the backreaction of the field onto the flow.

Methods. We use a high-resolution finite-volume ideal MHD code to perform 2D and 3D local simulations of hydromagnetic shear flows, both for idealized systems and simplified models of merger flows.

Results. In 2D, the magnetic field is amplified on time scales of less than $0.01 \mathrm{~ms}$ until it reaches locally equipartition with the kinetic energy. Subsequently, it saturates due to resistive instabilities that disrupt the Kelvin-Helmholtz unstable vortex and decelerate the shear flow on a secular time scale. We determine scaling laws of the field amplification with the initial field strength and the grid resolution. In 3D, the hydromagnetic mechanism seen in 2D may be dominated by purely hydrodynamic instabilities leading to less filed amplification. We find maximum magnetic fields $\sim 10^{16} \mathrm{G}$ locally, and rms maxima within the box $\sim 10^{15} \mathrm{G}$. However, due to the fast decay of the shear flow such strong fields exist only for a short period $(<0.1 \mathrm{~ms})$. In the saturated state of most models, the magnetic field is mainly oriented parallel to the shear flow for rather strong initial fields, while weaker initial fields tend to lead to a more balanced distribution of the field energy among the components. In all models the flow shows small-scale features. The magnetic field is at most in energetic equipartition with the decaying shear flow.

Conclusions. The magnetic field may be amplified efficiently to very high field strengths, the maximum field energy reaching values of the order of the kinetic energy associated with the velocity components transverse to the interface between the two neutron stars. However, the dynamic impact of the field onto the flow is limited to the shear layer, and it may not be adequate to produce outflows, because the time during which the magnetic field stays close to its maximum value is short compared to the time scale for launching an outflow (i.e., a few milliseconds).
\end{abstract}

Key words. magnetohydrodynamics (MHD) - instabilities - turbulence - stars: neutron - gamma-ray burst: general

\section{Introduction}

The merger of two neutron stars is considered the most promising scenario for the generation of short gamma-ray bursts (GRBs). After a phase of inspiral due to the loss of angular momentum and orbital energy by gravitational radiation, the merging neutron stars are distorted by their mutual tidal forces. Finally, they touch each other at a contact surface. Due to a combination of the orbital motion and the rotation of the neutron stars, the gas streams along that surface, the flow directions on either side of the surface being anti-parallel with respect to each other.

As a consequence of this jump in the tangential velocity, the contact surface is Kelvin-Helmholtz $(\mathrm{KH})$ unstable. Growing within a few milliseconds, the $\mathrm{KH}$ instability leads to the formation of typical KH vortices between the neutron stars. These vortices can modify the merger dynamics via the dissipation of kinetic into thermal energy. The generation of $\mathrm{KH}$ vortices is observed in actual merger numerical simulations (e.g., Oechslin et al. 2007).

The exponential amplification of seed perturbations can lead to very strong magnetic fields as shown by Price \& Rosswog (2006), and Rosswog (2007). These fields, in turn, can modify the dynamics of the instability described above, either already during its linear growth phase or, for weak fields, in the saturated state. Exerting stresses and performing work on the fluid, the magnetic field does lose part of its energy. Thus, the maximum attainable field strength is limited by the non-linear dynamics.

In their merger simulations, Price \& Rosswog (2006), and Rosswog (2007) observed fields exceeding by far $10^{15} \mathrm{G}$. Their numerical resolution, however, did not allow them to follow the detailed evolution of the $\mathrm{KH}$ instability in the non-linear phase. Thus, they could not draw any definite conclusions on the maximum strength of the field nor its back-reaction onto the fluid. They observed that the maximum field strength is a function of the numerical resolution: the better the resolution, the stronger becomes the field.

Performing numerical convergence tests, these authors did not find an upper bound for the field strength attainable in the magnetized KH instability. Thus, Price \& Rosswog (2006) discussed, based on energetic arguments, but not supported by simulation results, two different saturation levels: the field growth saturates when the magnetic energy density equals either the kinetic (kinetic equipartition) or the internal energy of the gas (thermal equipartition), corresponding to fields of the order 
of $10^{16} \mathrm{G}$ and $\sim 10^{18} \mathrm{G}$, respectively. From their simulations they were not able to identify the saturation mechanism applying to the KH instability in neutron-star mergers. Thus, we address this question here again using highly resolved simulations and independent numerical methods.

Most simulations of neutron-star mergers, including the ones by Price \& Rosswog (2006), and Rosswog (2007), are performed using smoothed-particle hydrodynamics (SPH) (Monaghan 1992). This free Lagrangian method is highly adaptive in space, and allows on to follow large density contrasts without "wasting" computational resources in areas of very low density. This property of SPH makes it highly advantageous for the problem of mergers. On the other hand, its relatively high numerical viscosity renders SPH inferior compared to Eulerian grid-based schemes for the treatment of (magneto)hydrodynamic instabilities and turbulence (Agertz et al. 2007). Moreover, the spatial resolution of most merger simulations is rather low, i.e., the reliability of their results concerning the details of the $\mathrm{KH}$ instability is limited.

A grid-based code such as ours is well suited for a study of flow instabilities and turbulence. Using it to simulate the entire merger event, however, is cumbersome due to the large computational costs required to cover the entire system with an appropriate computational grid. In spite of this fact, Giacomazzo et al. (2009) (see also Liu et al. 2008; Anderson et al. 2008) have performed full general-relativistic MHD simulations using vertexcentered mesh refinement to assess the influence of magnetic fields on the merger dynamics and the resulting gravitational waveform. But, as we shall show below, even their (presently world-best) grid resolution $(h \sim 350 \mathrm{~m})$ is still too crude to properly capture the disruptive dynamics after the $\mathrm{KH}$ amplification of the field. For comparison, we note here that our merger models employ a grid resolution of $h \sim 0.1 \mathrm{~m}$ in 2D (Sect. 6.2) and $h \sim 0.8 \mathrm{~m}$ in $3 \mathrm{D}$ (Sect. 6.3), respectively.

We performed a set of numerical simulations of the KH instability to understand the dynamics of magnetized shear flows and to draw conclusions on the evolution of merging neutron stars. The main issues we address in our study are motivated by two different, albeit related, intentions:

- We strive for a better understanding of the magnetohydrodynamic (MHD) KH instability. This includes the influence of numerical parameters such as the grid resolution on the dynamics, and generic properties of the saturation of the instability. We address these questions by a series of $d i$ mensionless models that use scale-free parameters as most previous studies focusing on the generic properties of the $\mathrm{KH}$ instability instead of a particular astrophysical application.

- We further want to verify the results of Price \& Rosswog (2006) and reassess their estimates of the saturation field strength. Hence, we consider the growth time of the instability that has to compete with the dynamical time scale of the merger event (a few milliseconds), the saturation mechanism, the saturation field strength, and generic dynamical features of supersonic shear flows. Our results should also allow us to reassess the findings of global simulations extending the ones performed by Price \& Rosswog (2006), e.g., the simulations by Anderson et al. (2008) and Liu et al. (2008).

To this end we utilize a newly developed multidimensional MHD code (Obergaulinger et al. 2009) that employs various explicit finite-volume algorithms, and that is particularly well suited for simulating instabilities and turbulent systems. As the code is based on Eulerian high-resolution methods instead of SPH as in Price \& Rosswog (2006), our results are complementary to theirs, serving as an independent check.

Since we are unable to simulate the entire merger event using fine resolution, we focus on the evolution of a small, representative volume around the contact surface. This local simulation allows us to concentrate on the dynamics of the magnetohydrodynamic KH instability. However, as our simulations lack the feedback from the dynamics occurring on scales larger than the simulated volume, its influence has to be mimicked by suitably chosen boundary conditions. We neglect neutrino radiation, and the gas obeys either an ideal-gas or a hybrid (barotropic and ideal-gas) equation of state (EOS), the latter serving as a rough model for nuclear matter.

This paper is organized as follows. We describe the physics of the magnetohydrodynamic $\mathrm{KH}$ instability in Sect. 2, and our numerical code in Sect. 3. We discuss the simulations addressing generic properties of the $\mathrm{KH}$ instability in two and three spatial dimensions in Sects. 4 and 5, respectively. The results applying to neutron-star mergers are given in Sect. 6. Finally, we present a summary and conclusions of our work in Sect. 7.

\section{The magnetohydrodynamic KH instability}

The KH instability leads to exponential growth of perturbations in a non-magnetized shear layer of a fluid of background density $\rho$ (e.g., Chandrasekhar 1961). If a plane-parallel shear layer extends over a thickness $d$, all modes with wavelengths $\lambda>d$ are unstable, shorter modes growing faster. After a phase of exponential growth, a stable KH vortex forms.

If the shear layer is threaded by a magnetic field of field strength, $b$, parallel to the shear flow (the $x$-direction in our models), magnetic tension stabilizes all modes, if the Alfvén number of the shear flow

$\mathrm{A} \equiv U_{0} / c_{\mathrm{A}}<2$

where $U_{0}$ and $c_{\mathrm{A}} \equiv \sqrt{b^{2} / \rho}$ are the velocity difference across the shear layer, and the Alfvén velocity, respectively. If the field is weaker, the instability can develop similarly to the non-magnetic case, but its growth and its non-linear saturated state are affected significantly (e.g., Frank et al. 1996; Jones et al. 1997; Jeong et al. 2000; Ryu et al. 2000).

A magnetic field perpendicular to the shear flow and the shearing interface (a $b_{y}$ field in our models) is sheared into a parallel $b_{x}$ field. Thus, the resulting flow dynamics is similar. A field orthogonal to the shear flow but parallel to the interface (a $b_{z}$ field in our models) acts mainly by adding magnetic pressure to the thermal one, thus modifying the dynamics of the KH instability only if its strength approaches or exceeds the equipartition field strength. Hence, we focus here on fields in the direction of the flow, only.

Depending on the field strength, the above authors identified three different regimes concerning the dynamics of the instability.

Rather strong fields with an Alfvén number slightly below 2 lead to non-linear stabilization. Too weak for stabilization initially, the field is amplified by the instability, and after less than one turnover of the $\mathrm{KH}$ vortex, it is strong enough to suppress further winding. The field, concentrated in thin sheets, annihilates in localized reconnection and, mediating the conversion of kinetic via magnetic into internal energy, destroys the vortex. The late phases of the evolution consist of a very broad transition layer between those parts of the fluid moving in opposite directions. The flow is almost entirely parallel to the initial shear 
layer, and no vortex is retained. The magnetic field has decreased strongly due to reconnection, and is still concentrated in sheetlike patterns.

Weaker fields give rise to disruptive dynamics. The amplification process takes longer to produce strong fields, i.e., the vortex survives several turnover times. The field is wound up in increasingly thin sheets, that eventually reconnect due to (numerical) resistivity. Afterwards the dynamics is similar to the previous case: the vortex is disrupted, leading to a broad laminar transition region threaded by filamentary magnetic fields.

For even weaker fields one encounters the flow regime of dissipative dynamics. Even after a long phase of amplification, the field is still too weak to affect the flow. Reconnection occurs, but due to the weakness of the involved fields, it leads only to a gradual conversion of kinetic into internal energy. The global topology of the flow does not change as in the previous cases, and the vortex exists throughout the evolution. Its velocity decreases slowly as kinetic energy is extracted from the vortex.

We note that the transition between these three dynamic regimes is not sharp. In particular, it is not possible to define a threshold Alfvén number separating disruptive and dissipative dynamics.

Further complications arise in three spatial dimensions. Here, the KH vortex can be disrupted even without the presence of a magnetic field by purely hydrodynamic instabilities (Ryu et al. 2000), and the effects of a magnetic field overlay with those of the non-magnetic instabilities.

\section{Numerical methods}

We use a newly developed high-resolution code to solve the equations of ideal (Newtonian) MHD (Einstein's summation convention applies),

$$
\begin{aligned}
\partial_{t} \rho & +\nabla_{j}\left[\rho v^{j}\right]=0, \\
\partial_{t} p^{i} & +\nabla_{j}\left[p^{i} v^{j}+P_{\star} \delta^{i j}-b^{i} b^{j}\right]=f^{i}, \\
\partial_{t} e_{\star} & +\nabla_{j}\left[\left(e_{\star}+P_{\star}\right) v^{j}-b^{i} v_{i} b^{j}\right]=f_{j} v^{j}, \\
\partial_{t} \boldsymbol{b} & =-c \boldsymbol{\nabla} \times \boldsymbol{E}, \\
\nabla_{j} b^{j} & =0,
\end{aligned}
$$

where the mass density, momentum density, velocity, and totalenergy density of the gas are denoted by $\rho, \boldsymbol{p}, \boldsymbol{v}$, and $e_{\star}$, respectively; $\boldsymbol{b}$ is the magnetic field. The total-energy density and the total pressure, $P_{\star}$, are composed of fluid and magnetic contributions: $e_{\star}=\varepsilon+\rho \boldsymbol{v}^{2} / 2+\boldsymbol{b}^{2} / 2$, and $P_{\star}=P+\boldsymbol{b}^{2} / 2$, where $\varepsilon$ and $P=P(\rho, \varepsilon, \ldots)$ are the internal energy density and the gas pressure, respectively. The electric field, $\boldsymbol{E}$, is given by $\boldsymbol{E}=-(\boldsymbol{v} / c) \times \boldsymbol{b}$ with $c$ being the speed of light in vacuum. The external force, arises from gravity, i.e, $\boldsymbol{f}=\boldsymbol{f}_{\mathrm{G}}=-\rho \boldsymbol{\nabla} \Phi$, where $\Phi$ is the gravitational potential.

The above equations are implemented into our code in their finite-volume form. We use Eulerian high-resolution shockcapturing methods for their solution (see, e.g., LeVeque 1992). To reconstruct the zone interface values of variables defined as volume averages over grid zones, we use high-order algorithms of one of the following types:

- Piecewise-linear reconstruction using total-variation diminishing (TVD) methods (Harten 1983). While formally 2nd order accurate in smooth parts of the flow and away from local extrema, these methods achieve a stable representation of discontinuities by reverting to 1 st-order accurate piecewiseconstant reconstruction. The accuracy of the scheme depends on its slope limiter for which different choices are possible, e.g., the Minmod, the van Leer, or the MC (monotonized central) limiters.

- The class of weighted essentially non-oscillatory (WENO) algorithms (Liu et al. 1994) offer a way of constructing schemes of arbitrarily high order of accuracy. In these methods, an interpolant for a variable at a given point in space (e.g., a zone interface) is constructed from a number of candidate polynomials by maximizing a measure of the smoothness of these polynomials. In our scheme, based on the one described by Levy et al. (2002), we use three candidate parabolas, leading to a nominal order of accuracy of 4 .

- Suresh \& Huynh (1997) use a generalization of the TVD criterion to construct high-order monotonicity-preserving (MP) schemes. The new MP stability and accuracy constraints do not lead to the clipping of extrema in smooth regions of the flow that is innate to the TVD criteria. Thus, they allow for a higher accuracy in smooth flows while retaining stability close to discontinuities. Suresh \& Huynh (1997) give MP schemes of formally 5 th, 7 th, and 9 th order that we implemented in our code.

We compute the fluxes of the MHD equations from the reconstructed interface states using approximate Riemann solvers. Titarev \& Toro (2005) and Toro \& Titarev (2006) developed multi-stage (MUSTA) Riemann solvers that are built on a combination of predictor and corrector steps using simple approximate Riemann solvers. These solvers do not require a computationally expensive decomposition of the MHD state into characteristic variables, yet they achieve an accuracy comparable to exact solvers.

In MHD simulations, it is important to use a numerical scheme that keeps the magnetic field divergence-free. To this end we employ in our code the constraint-transport (CT) scheme of (Evans \& Hawley 1988) that uses a spatial discretization of the magnetic field consistent with the curl operator in the induction equation, leading to a staggering of the collocation points of $\boldsymbol{b}$ with respect to those of the hydrodynamic variables $\rho, \boldsymbol{p}$, and $e_{\star}$. According to the definition of $\boldsymbol{b}$ the electric field, $\boldsymbol{E}$, is defined as the average over the zone edges. The staggering of $\boldsymbol{b}$ requires interpolations between the staggered grids (to obtain, e.g., the Maxwell stress $b^{i} b^{j}$; see Eq. (3)), and special care has to be taken in the computation of the electric field from the (zone-centered) velocity and the (zone-interface) magnetic field. Various implementations of the CT scheme have been devised that differ mainly in the way the magnetic stress and electric field are calculated. Of these, our implementation resembles most closely the recently developed upwind-CT schemes (Londrillo \& del Zanna 2004; Gardiner \& Stone 2005, 2008). We obtain $\boldsymbol{E}$ from the zone interface values of the velocity and the magnetic field that are both computed by the (MUSTA) Riemann solver. This guarantees that the electric field is consistent with the solution of the Riemann problem.

Our code is written in FORTRAN 90 and parallelized for shared or distributed memory computers using the OpenMP or MPI programming paradigm, respectively. The code successfully passed various standard tests including MHD shock tube problems (e.g., the ones published by Ryu \& Jones 1995), the propagation of MHD waves, and some multi-dimensional flow problems such as the Orszag-Tang vortex (Orszag \& Tang 1979). These tests demonstrate the stability and accuracy of the code in handling flows involving discontinuities and turbulent structures. According to the results of the wave-propagation tests, the order of accuracy of the code is $2,3.3$, and 4.1 for 
piecewise-linear, MP, and WENO reconstruction, respectively (Obergaulinger 2008). The code has also been used to study the magneto-rotational instability (MRI) in core collapse supernovae (Obergaulinger et al. 2009).

The simulations reported in this paper were performed with MP reconstruction based on 5th-order polynomials (the MP5 method), and the MUSTA solver derived from the HLL Riemann solver. This reconstruction method represents a good trade-off between accuracy and computational costs. Methods based on higher-order polynomials increase the accuracy of the code, but at the expense of a larger stencil, reducing the efficiency of the parallel code, since the number of ghost zones that have to be communicated among different processors is larger. The same adverse effect on the computational efficiency can be observed when comparing our WENO reconstruction to MP5.

\section{The KH instability in 2D planar magnetized shear flows}

We performed a set of two dimensional simulations to study the properties of the $\mathrm{KH}$ instability in 2D planar magnetized shear flows. These simulations allow us to validate our numerical tool and to assess the significance of results obtained in simulations aiming at an understanding of the $\mathrm{KH}$ instability in neutron-star mergers.

As we shall show below we reproduce, but also extend the results obtained by Frank et al. (1996), Jones et al. (1997), Baty et al. (2003), and Keppens et al. (1999) which are summarized in Sect. 2.

We consider both subsonic and supersonic 2D planar shear flows in the $x-y$ plane in $x$-direction with an initial velocity profile given by (note that all numerical values are given in dimensionless code units in the following!)

$$
\left(v_{x}, v_{y}\right)^{T}=\left(v_{0} \tanh \frac{y}{a}, 0\right)^{T}
$$

where $U_{0}=2 v_{0}$ is the shear velocity, and $a$ is a length scale characterizing the width of the shear flow. The background density and pressure are uniform, and the thermodynamic properties of the fluid are described by an ideal-gas EOS with an adiabatic index $\Gamma$,

$P=(\Gamma-1) \varepsilon$,

where $\varepsilon=e_{\star}-\frac{1}{2} \rho \boldsymbol{v}^{2}-\frac{1}{2} \boldsymbol{b}^{2}$ is the internal energy density of the fluid. Initially, a uniform magnetic field $\boldsymbol{b}(t=0)=\left(b_{x}^{0}, . b_{y}^{0}\right)^{T}$ threads the shear layer.

To trigger the KH instability we perturb the shear flow by a transverse velocity

$v_{y}(t=0)=v_{y}^{0} f(y) \sin \left(k_{x} x\right)$,

where $f$ (with $f(y) \in[0,1]$ ) is a function localized at the shearing interface, i.e., it vanishes beyond a distance $a^{\prime}$ from the interface. We set $a^{\prime}=4 a$ here. The maximum perturbation velocity, $v_{y}^{0}$, is typically a factor $10^{6 \ldots 8}$ smaller than the shear velocity. To test the influence of the form of the perturbations, we also simulated some models (both magnetic and non-magnetic ones) with random perturbations which do not select a priori a single sinusoidal unstable mode (see below).

Finally, we introduce the volume-averaged kinetic energy densities

$e_{\mathrm{kin}}^{i} \equiv \frac{1}{\mathcal{V}} \int \mathrm{d} \mathcal{V} \frac{1}{2} \rho v_{i}^{2}$,

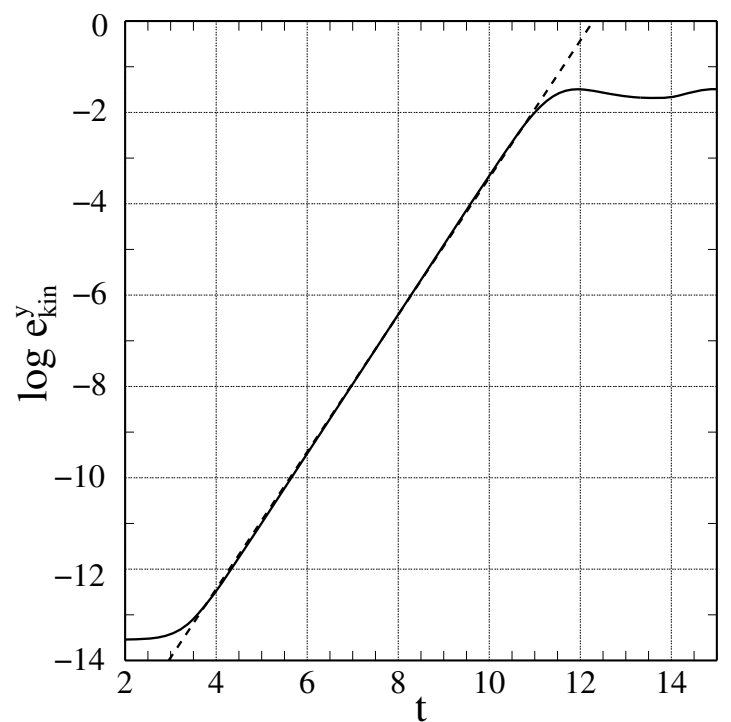

Fig. 1. Linear growth phase of the KH instability in model grw-3. The solid black line shows the volume-averaged kinetic energy density $e_{\mathrm{kin}}^{y}$, as a function of time $t$. The dashed line gives the theoretical growth rate.

and volume-averaged magnetic energy densities

$e_{\mathrm{mag}}^{i} \equiv \frac{1}{\mathcal{V}} \int \mathrm{d} \mathcal{V} \frac{1}{2} b_{i}^{2}$

with $i \in x, y, z$. These quantities will be useful for the following discussion.

\subsection{Linear growth}

Our code reproduces the growth rate of the $\mathrm{KH}$ instability very accurately. To demonstrate this we recalculated some of the models studied by Keppens et al. (1999) (models grw- $n$ in Table A.1). The growth rates for these models are either given in Keppens et al. (1999), or can be obtained from the figures of Miura \& Pritchett (1982).

The models have a uniform background density $\rho_{0}=1$, and a uniform background pressure $P_{0}$. We impose open boundary conditions in the transverse $(y)$ direction, periodic ones in $x$ direction, and vary the value of the shear velocity, the width of the shear layer, and the grid resolution.

We derive growth rates, $\Gamma_{\text {num }}$, from the exponential growth of $e_{\text {kin }}^{y}$, and compare these to the values, $\Gamma_{\mathrm{MP}}$, given by Miura $\&$ Pritchett (1982) and Keppens et al. (1999), respectively. We note in this respect that $e_{\mathrm{kin}}^{y}(t) \propto v_{y}^{2} \propto(\exp \Gamma t)^{2}$ (see Eq. (10)) grows at twice the rate of the KH instability. The agreement between the theoretical predictions and our numerical results is, in general, very good (see Table A.1 and Fig. 1).

After the initial phase of exponential growth, a roughly circular vortex develops in the perturbed non-magnetized shear layer which should be eventually dissipated by (numerical) viscosity. However, this process is very slow for our models (we see no sign of dissipation until the end of our simulations), as the numerical viscosity of our code is very low.

The formation of a single KH vortex rather than of a multitude of small vortices is not an artifact of the form of the initial perturbation (Eq. (9)). To demonstrate this, we simulated a non-magnetic model with random rather than sinusoidal perturbations of the transverse velocity with an amplitude of $10^{-6}$ of 


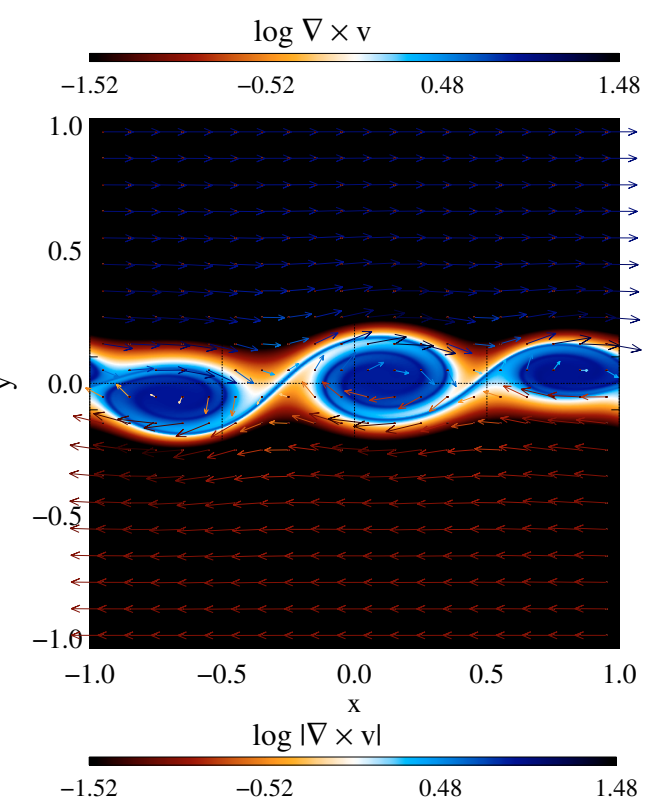

(a)

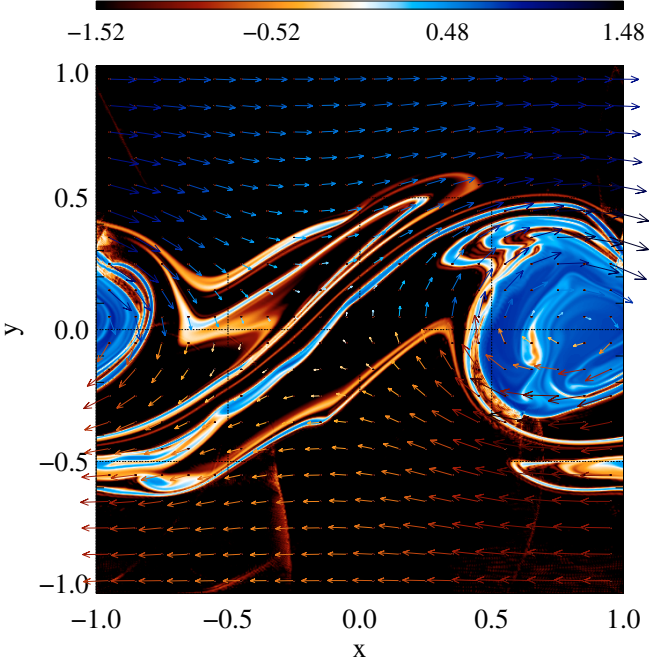

Fig. 2. Logarithm of the modulus of the flow vorticity and the velocity field (vectors) of a non-magnetic model with $\mathrm{M}=1$ and a random perturbation at $t=16($ panel a)), and $t=25.5$ (panel b)), respectively.

the shear velocity (see Fig. 2 for two snapshots of the model simulated with $1024^{2}$ zones at $t=16$ and $t=25.5$ (panels (a) and (b), respectively). Initially, three small $\mathrm{KH}$ vortices develop (panel (a)), but after two subsequent mergers of these vortices, only one large vortex remains (panel (b)), resembling closely the flow field of a model with sinusoidal perturbation. Due to this evolution towards a single large-scale vortex, we focus on models with sinusoidal perturbations in the following ${ }^{1}$.

\subsection{Non-magnetic models}

We simulated a set of non-magnetic models (summarized in Table A.2) to study the influence of the box size and boundary conditions on the evolution of transonic and supersonic ( $\left.\mathrm{M}=U_{0} / c_{\mathrm{S}} \geq 1.8\right)$ shear flows. As noted by Miura \& Pritchett (1982), there is no growing mode for a $M \geq 2$ shear flow, but in models with closed boundaries we find nevertheless a growing

1 Without elaborating in more detail, we note that a similar result holds for magnetized models. instability whose growth mechanism is, however, different (see below).

We first consider models with $\mathrm{M}=1.8$. For these models the instability grows faster when the vertical domain size is enlarged, and open boundaries yield larger growth rates than reflecting ones. The reason for this behavior is that the instability affects a larger region of the flow than in the case of slow shear flows. To demonstrate this we compare models HD2o-1 and HD2o-1-s that differ only in the size of the computational domain in $y$-direction: $y \in[-1 ; 1]$ and $y \in[-0.25 ; 0.25]$ for models HD2o-1 and HD2o-1-s, respectively. According to Fig. 3 the volume-averaged kinetic energy density, $e_{\text {kin }}^{y}$, grows faster and leads to much larger values in model HD20-1 than in model HD2o-1-s. Furthermore, in model HD2o-1-s the growth of $e_{\text {kin }}^{y}$ shows superimposed oscillations. In both models waves are created at the shear layer which travel outwards in $y$-direction carrying (transverse) kinetic energy. If the waves are allowed to travel over a sufficiently long distance $\delta y$ (which is the case for model HD2o-1), they steepen into shock waves when the fluid velocity exceeds the sound speed. The shocks propagate mainly in $x$-direction, advected by the shear flow. Kinetic energy is dissipated into internal one in these shocks, and the flow develops a vortex-like structure. If the boundaries of the computational domain are too close to the shear layer, the waves leave the domain before they can affect the flow, i.e., the growth rate is reduced. Each time a wave leaves the computational domain, it carries away kinetic energy giving rise to the oscillations of $e_{\text {kin }}^{y}$ visible in Fig. 3.

For an intermediate domain size of $y \in[-0.5 ; 0.5]$ (model HD2o-1-s), we find despite the absence of oscillations a smaller growth rate than for models HD2o-1 $(y \in[-1 ; 1])$ and HD2o$1-1(y \in[-2 ; 2])$, respectively. The boundaries are sufficiently close to the shear layer to affect the growth of the instability. Saturation occurs by the same mechanism as in case of a larger domain, namely by the development of shock waves.

The distance the waves travel in transverse direction increases with increasing Mach number of the shear flow. For $\mathrm{M}=1$ the waves are contained essentially in the region $y \in$ $[-0.25 ; 0.25]$ (a version of model grw-3 simulated on a smaller grid of $200 \times 100$ zones covering a domain of $[-0.5 ; 0.5] \times$ $[-0.25 ; 0.25]$ does not show oscillation of $e_{\text {kin }}^{y}$ ). For the same reason the evolution does not depend on whether one imposes reflecting or open boundary conditions (compare models HD2r0 in Table A.2 and grw-3 in Table A.1). Thus, to encounter a rapidly growing instability in a fast shear flow, one has to simulate a sufficiently large domain, or alternatively to use reflecting boundaries in $y$-direction. For $\mathrm{M}=1.8$, open and closed models (i.e., models where open or reflecting boundaries are imposed) agree in their growth rates if simulated on a sufficiently large domain. However, when the extent of the computational domain is small in the transverse direction $\left(l_{y}=0.5\right)$, we observe a destabilization of closed models: the growth rate of the closed model HD2r-1-s exceeds that of the corresponding open model HD2o1 -s by a factor of $\approx 3.5$. Furthermore, closed models exhibit a phase of exponential and oscillatory growth of $e_{\text {kin }}^{y}(t)$ even when $\mathrm{M}>2$, whereas open models are stable.

In the $\mathrm{KH}$ saturated state the flow consists of a dominant vortex for shear flows of moderate Mach numbers. At large Mach numbers and when the growth of the instability is mediated mainly by shock waves, the flow is characterized by a rather thin and clearly delimited transition layer oriented along the initial discontinuity (at $y=0$ ). This layer is surrounded by two regions of anti-parallel flows. 


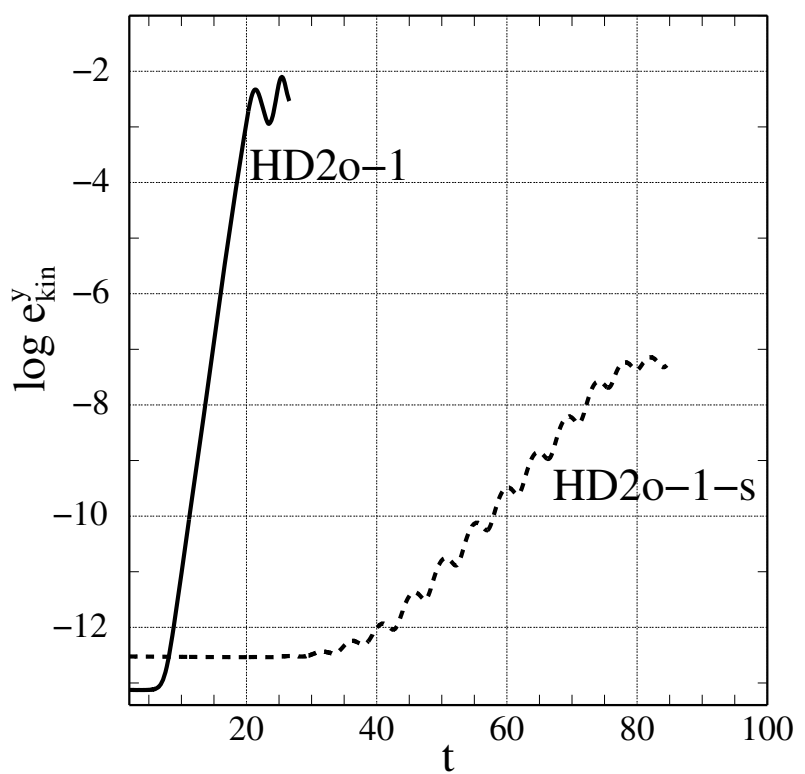

Fig. 3. Volume averaged kinetic energy density $e_{\text {kin }}^{y}$ of models HD2o-1 (solid line; $l_{y}=2$ ) and HD2o-1-s (dashed line; $l_{y}=0.5$ ) as a function of time illustrating the influence of the size of the vertical extent of the computational domain $l_{y}$.

The shocks created at the supersonic shear layer are initially oblique, but eventually become planar shocks parallel to the $y$-direction. This process happens earlier close to $y=0$. The vertical extent of the planar shock structures varies from a fraction of the vertical domain size to almost the whole computational box. When the propagation of the shocks is restricted in $y$-direction, the fluid tries to avoid these by sliding along the vertical direction. Thus, the planar shocks very efficiently convert $x$ - into $y$-kinetic energy.

\subsection{Intermediate and weak fields}

Sufficiently strong magnetic fields (Alfvén number $A \leq 2$; see Eq. (1)) stabilize the flow according to linear stability analysis. We indeed observe this stabilization in simulations of both subsonic and supersonic strongly magnetized shear flows. In the following, we thus focus on the more interesting case of intermediate and weak initial fields, which according to Frank et al. (1996) can give rise to disruptive and dissipative dynamics, respectively. The models we describe in this section were computed using a grid with $l_{x} \times l_{y}=2 \times 2$ and reflecting boundary conditions in $y$-direction. We simulated shear flows with $U_{0}=1$, and varied the Mach number of the flow by setting the pressure either to $P_{0}=0.6$ or $P_{0}=0.0375$ corresponding to Mach numbers of $M=1$ and $M=4$, respectively. The adiabatic index of the gas was $\Gamma=4 / 3$.

\subsubsection{Intermediate fields}

For $A=2.5$, we find, in agreement with Frank et al. (1996), nonlinear stabilization. The magnetic field is amplified during the linear phase, and the magnetic tension becomes eventually sufficiently strong to prevent further bending of the field lines. Thus, the formation of a $\mathrm{KH}$ vortex is suppressed. Instead, the velocity and the magnetic fields remain essentially aligned with each other and the shear layer developing only small $y$-components. After the end of linear growth a broad shear layer develops inside which the magnetic field has a sheet-like structure.

If the magnetic field strength is reduced further $(A=5)$, we observe a linear growth of the KH instability, and the formation of a $\mathrm{KH}$ vortex. The overturning vortex continues to amplify the field until it becomes eventually so strong that it resists further bending, i.e. the instability saturates in the non-linear phase. The magnetic energy, which grows exponentially during the linear phase, reaches a maximum, and then gradually declines back to almost its initial value.

It is important to note that although we are evolving the equations of ideal (i.e., non-resistive) MHD numerical resistivity is present and acts similar as a physical resistivity. Hence, reconnection of field lines and dissipation of magnetic energy into internal energy occurs. Though being a purely numerical effect, this dissipation mimics a physical process: in ideal MHD (or for exceedingly large magnetic Reynolds number $R e_{m}$ ), energy is transferred to ever smaller length scales by a turbulent cascade. When the cascade reaches the scale set by the grid resolution, the physics is no longer appropriately represented by the discretized magnetic field. Instead, the unresolved (sub-grid) magnetic energy is assigned to the internal energy. Hence, numerical resistivity (like numerical viscosity) acts as an unspecific sub-grid model for unresolved dynamics.

As a result of numerical resistivity, our models show the dynamics discussed by Jones et al. (1997): the emergence of coherent flow and field structures, and their subsequent disruption in intense reconnection events whereby kinetic energy is efficiently converted into internal energy. As a consequence, the kinetic energy decreases more strongly than in the non-magnetic case, and the flow barely resembles a $\mathrm{KH}$ vortex at the end of the simulation. Instead, we find a broad transition layer that is embedded into two anti-parallel flows and that contains thin magnetic flux sheets. The flow is rather laminar than turbulent, with elongated streaks of gas and field stretching across the computational domain.

\subsubsection{Weak fields}

Overview: Models with a weak initial magnetic field show disruptive or dissipative dynamics (Jones et al. 1997). In both regimes, a KH vortex develops. The magnetic field forms thin flux sheets while it is wound up by the vortex. If two flux sheets of opposite polarity come to lie close to each other, they suffer the resistive tearing-mode instability which leads to the reconnection of field lines of different orientation and the conversion of magnetic into thermal energy. Since the magnetic energy was previously amplified at the cost of the kinetic energy, the tearing modes act essentially as a catalyst facilitating the dissipation of kinetic into internal energy. This behavior characterizes the dissipation regime, while in the disruption regime another effect comes into play: the magnetic field eventually becomes sufficiently strong to disrupt the vortex leaving behind a broad transition layer where turbulent flow and magnetic fields decay slowly. The dynamics of the flow and the magnetic field are highly coupled since the field is dominated by flux sheets where the velocity and the magnetic field are strongly aligned, reminiscent of the Alfvén effect in MHD turbulence (Iroshnikov 1964; Kraichnan 1965). Accordingly, we also find near equipartition between the transverse magnetic and kinetic energy densities (see the disruption models below). 
M. Obergaulinger et al.: Local simulations of the magnetized Kelvin-Helmholtz instabilityin neutron-star mergers

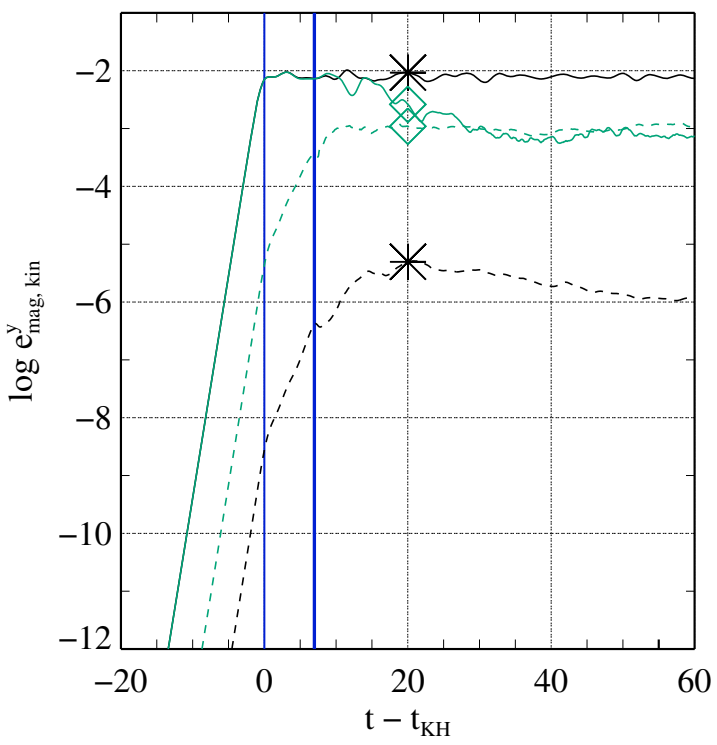

Fig. 4. Volume averaged transverse kinetic (solid) and magnetic (dashed) energy densities $e_{\mathrm{kin}}^{y}$ and $e_{\mathrm{mag}}^{y}$ versus time for models with an initial Mach number $M=1$, and Alfvén numbers $A=125$ (green, diamond) and $A=5000$ (black, asterisk), respectively. Both models were computed using a grid of $2048^{2}$ zones. The blue vertical lines indicate the end of the $\mathrm{KH}$ phase, $t_{\mathrm{KH}}$, and of the kinematic phase, respectively.

The evolution of the simulated weak-field models (summarized in Table A.3) consists of three distinct phases:

- Linear KH growth phase: initial perturbations of both velocity and magnetic field grow exponentially until a $\mathrm{KH}$ vortex forms.

- Kinematic field amplification phase: magnetic field is wound up by the secularly evolving $\mathrm{KH}$ vortex.

- Dissipation/disruption phase: KH vortex looses its energy due to magnetic stresses and resistive effects.

We discuss these three phases and the transitions between them in more detail in the following. The phases can be distinguished best on the basis of the evolution of the transverse kinetic and magnetic energy densities $e_{\mathrm{kin}}^{y}$ and $e_{\mathrm{mag}}^{y}$, respectively (see Eqs. (10) and (11); Fig. 4). For this purpose, we consider a pair of prototype models, with initial Mach number $M=1$, and Alfvén numbers $A=125$ and $A=5000$, respectively, computed on a grid of $2048^{2}$ zones.

KH growth phase: Early on during the evolution the seed perturbations imposed on the initial shearing profile are amplified exponentially, but the magnetic field remains too weak to affect the evolution. When the exponential growth of the KH instability terminates, the total magnetic energy has grown by about a factor 1.4 in all models, the contribution of the transverse field component $b^{y}$ amounting to about $10 \%$. Due to the persisting weakness of the magnetic field the growth rate of the instability and the flow structure after the end of the KH growth phase are the same as those without any field.

When the $\mathrm{KH}$ instability saturates with the formation of a KH vortex (see Fig. 6 for a model with $A=125$ ), the growth of the transverse kinetic energy ceases, too (Fig. 4). Density, pressure, sound speed, and magnetic field strength possess a minimum at the center of the vortex, and the magnetic field is wound up into a long thin sheet surrounding the vortex. These findings

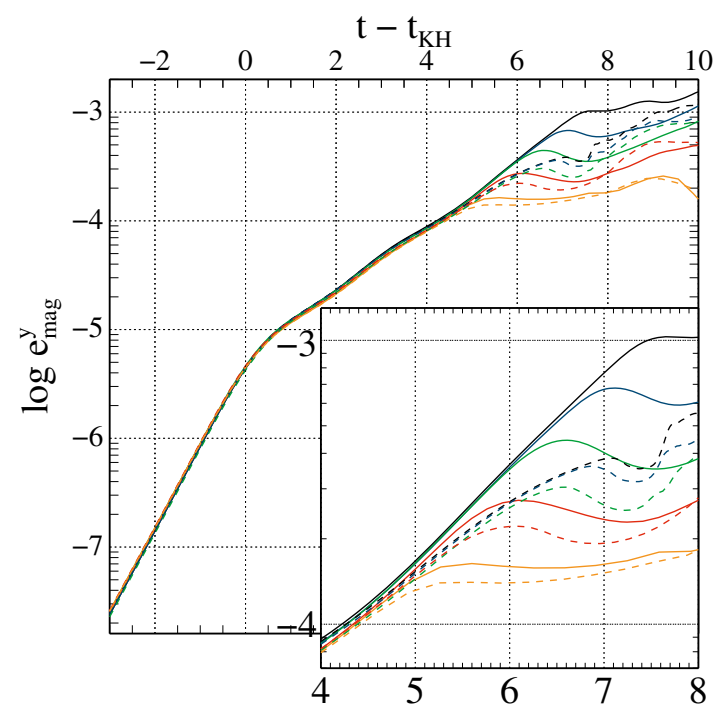

Fig. 5. Growth of the volume-averaged turbulent (transverse) magnetic energy density $e_{\text {mag }}^{y}$ (see Eq. (11)) with time for two different models simulated at five different grid resolutions. At time zero the ratio of $e_{\text {kin }}^{y}$ and the volume-averaged total magnetic energy density is maximal, i.e. this moment corresponds to $t_{\mathrm{KH}}$. The solid and dashed lines refer to a model with initial Alfvén numbers $A=5000$ and 125, respectively. Note that the $f$ values of the former model are scaled by the factor $(5000 / 125)^{2}$. Orange, red, green, blue, and black lines refer to simulations with $256^{2}, 512^{2}, 1024^{2}, 2048^{2}$, and $4096^{2}$ zones, respectively. The insert shows a magnified view of the late evolution.

hold for the models with $A=125$ and $A=5000$, respectively. Fig. 5 shows that the growth rate of the instability (the slope of the curves) is independent of the grid resolution and the initial field strength for $t-t_{\mathrm{KH}}<0$.

Kinematic amplification phase: After saturation of the essentially hydrodynamic $\mathrm{KH}$ instability, $e_{\text {kin }}^{y}(t)$ exhibits small oscillations about a constant value. The initial shearing interface, wound up several times by the overturning vortex, has become a thin fluid layer separating flow regions of opposite velocities Fig. 6). The magnetic sheet is being stretched by the overturning vortex giving rise to an exponential amplification of the field (instead of a linear one by winding), as the growth rate due to stretching depends on the field strength itself. In spite of the growing magnetic field the flow structure as well as the kinetic and internal energies of the fluid show only minor changes throughout the entire kinematic amplification phase.

To understand the amplification of the magnetic field in detail we consider the sources and sinks of magnetic energy. From the scalar product of $\partial_{t} \boldsymbol{b}$ (given by the induction equation) and the magnetic field, $\boldsymbol{b} \cdot \partial_{t} \boldsymbol{b}$, one can derive the equation for the evolution of the total energy density of the magnetic field, $e_{\text {mag }}$, which has the form of an advection equation with source terms,

$\partial_{t} e_{\mathrm{mag}}+\boldsymbol{\nabla}\left(e_{\mathrm{mag}} \boldsymbol{v}\right)=s_{\mathrm{mag}}$

The source term,

$s_{\mathrm{mag}}=-e_{\mathrm{mag}} \boldsymbol{\nabla} \cdot \boldsymbol{v}+b^{x} b^{y}\left(\partial_{y} v^{x}-\partial_{x} v^{y}\right)$,

consists of a compression term proportional to the divergence of the velocity field, and a shear term proportional to the curl of the 


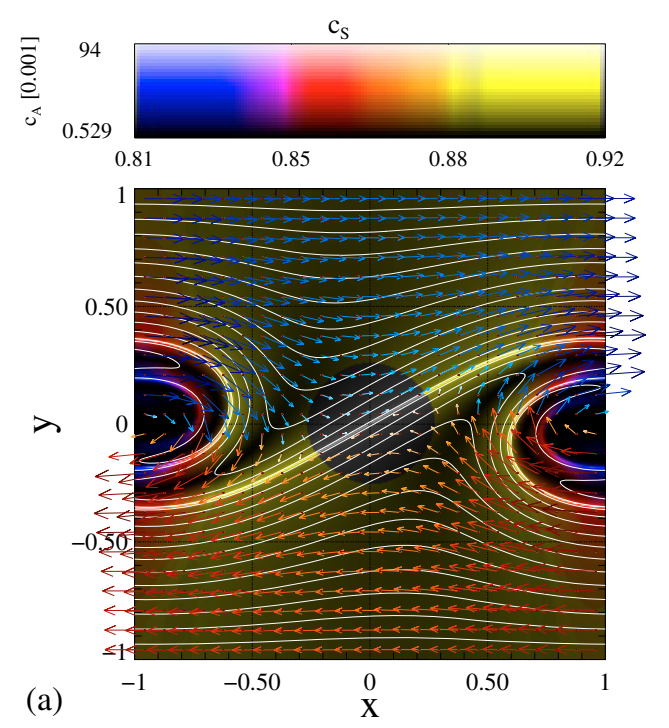

Fig. 6. Snapshot of a model with initial Mach number $M=1$ and Alfvén number $\mathrm{A}=125$ computed on a grid of $2048^{2}$ zones shortly after the end of the KH growth phase. The hue gives the sound speed, $c_{\mathrm{s}}$, and the brightness of the colors the Alfvén velocity, $c_{\mathrm{A}}$, respectively. Magnetic field lines and flow velocity vectors are shown, too. The latter are colorcoded according to the size of the $x$-component of $\boldsymbol{v}$, reddish and bluish colors corresponding to matter flowing to the left and right, respectively.

velocity field. The sum of both terms (i.e., the source term) is negative, when the magnetic field does work on the fluid.

The evolution of $e_{\text {mag }}$ is exemplified in Fig. 7 for a model with $A=125$. As the fluid is nearly incompressible in our models, the first term on the r.h.s. of Eq. (13) is small, and field amplification (bluish areas) occurs predominantly by stretching. As there is no back-reaction onto the flow, the volume-averaged transverse magnetic energy density grows exponentially with time. Stretching mainly happens in the thin flux sheet passing through the origin of the grid, and to a lesser extent in the flux sheets located closer to the center of the vortex. There even a small reduction of $e_{\text {mag }}$ can be observed (see Fig. 7). The volume integral of the source term over the entire computational domain is positive, i.e., the magnetic energy of the models is increasing.

Because field amplification is mediated by a well resolved, rather smooth flow, the growth rate of the turbulent magnetic energy density $e_{\text {mag }}^{y}$ is independent of the grid resolution during the kinematic amplification phase $\left(0 \leq t-t_{\mathrm{KH}} \lesssim 5\right.$; see Fig. 5). Models with $M=0.5$, but otherwise identical initial conditions and grid resolution, show a slower growth of the field (see Table A.3). As $e_{\text {kin }}^{y}$ (monitoring the turnover velocity of the vortex) shows small variations with time during the kinematic amplification phase (see Fig. 4), the growth rate varies slightly, too (note the variation of the slope in Fig. 5 for $2 \lesssim t-t_{\mathrm{KH}} \lesssim 4$ ).

The evolution of the turbulent magnetic energy density after the end of the kinematic amplification phase depends strongly on the grid resolution and the initial field strength (Fig. 5). Comparing the results for the models with $\mathrm{A}=125$ and $\mathrm{A}=$ 5000 we conclude that the growth of the turbulent magnetic energy density is less for models with a stronger initial field than for those with a weaker initial field at the same grid resolution.

For the model with $\mathrm{A}=125$ the magnetic field eventually reaches locally (within a factor of a few) equipartition strength, i.e., magnetic stresses start to change the flow. In the model with the lower initial Alfvén velocity (i.e., larger Alfvén number), the magnetic field remains, in spite of a larger amplification, too weak to cause such an effect.

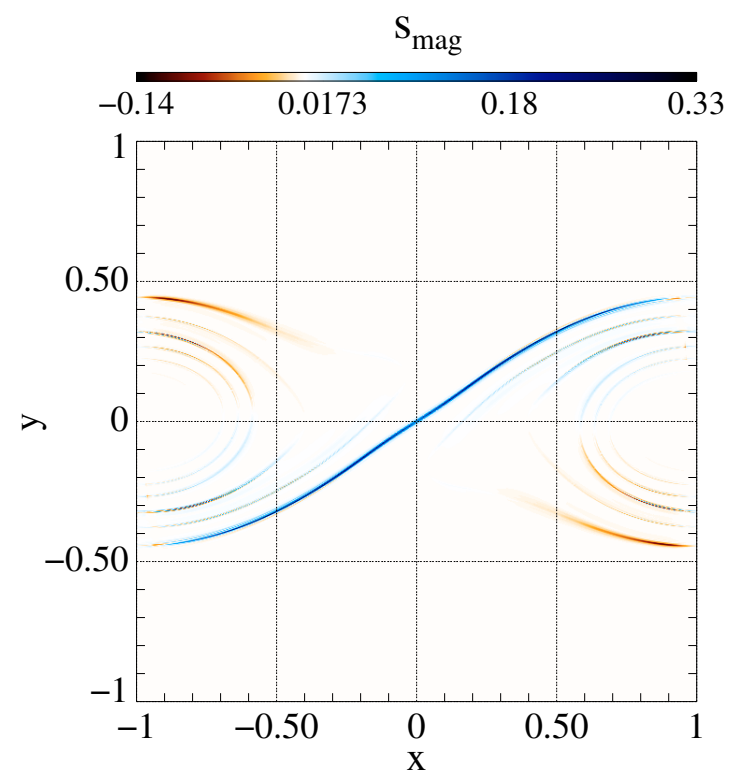

Fig. 7. Snapshot of the source term of the total magnetic energy density (Eq. (13)) for the model shown in Fig. 6 taken during the kinematic amplification phase. Reddish (bluish) colors show regions where the total magnetic energy density increases (decreases).

To quantify the amount of amplification of the magnetic field occurring during the kinematic amplification phase we introduce the field amplification factor

$f_{\text {kin }}=\mathrm{M}_{x y}\left(t=t_{\text {kin }}\right) / \mathrm{M}_{x y}\left(t=t_{\mathrm{KH}}\right) \equiv \mathrm{M}_{x y}^{\mathrm{kin}} / \mathrm{M}_{x y}^{\mathrm{KH}}$

defined as the ratio of the off-diagonal volume-integrated Maxwell stress component $\mathrm{M}_{x y}$ at the end of the kinematic amplification phase and at the end of the KH growth phase.

When plotting $f_{\text {kin }}$ as a function of grid resolution and initial Alfvén number we find that our models populate the lower right region (shaded in gray) in both diagrams (Fig. 8). Both for a given grid resolution and initial Alfvén number, $f_{\text {kin }}$ converges towards a maximum value with increasing initial Alfvén number (Fig. 8, left panel), and increasing grid resolution (Fig. 8, right panel). This convergence is also obvious from the graph of $f_{\text {kin }}\left(m_{X}\right)$ for $\mathbf{A}=$ const. (Fig. 8, left panel); note that for large values of $A$ even our finest the grid spacing was not yet sufficient to show the flattening of $f_{\text {kin }}\left(m_{X}\right)$.

The panels further show that the weaker (larger) the initial field (the value of $\mathrm{A}$ ), the higher is the amplification factor $f_{\mathrm{kin}}$ achievable during the kinematic amplification phase. The upper border of the gray shaded regions is approximately given by the power laws $m_{x}^{7 / 8}$ and $\mathrm{A}^{3 / 4}$, respectively.

To explain these results and to quantify the effects of the grid resolution, we define a characteristic length scale of variations of the magnetic field

$l_{b}=\frac{|\boldsymbol{b}|}{|\boldsymbol{\nabla} \times \boldsymbol{b}|}$,

where the denominator is proportional to the current density. Initially infinite (the initial magnetic field is curl free), $l_{b}$ decreases during the $\mathrm{KH}$ growth and the kinematic amplification phases.

Due to flux conservation, the amplification of the field occurring mainly in flux sheets goes along with a decrease of the width of the sheets orthogonal to the magnetic field, which is roughly 


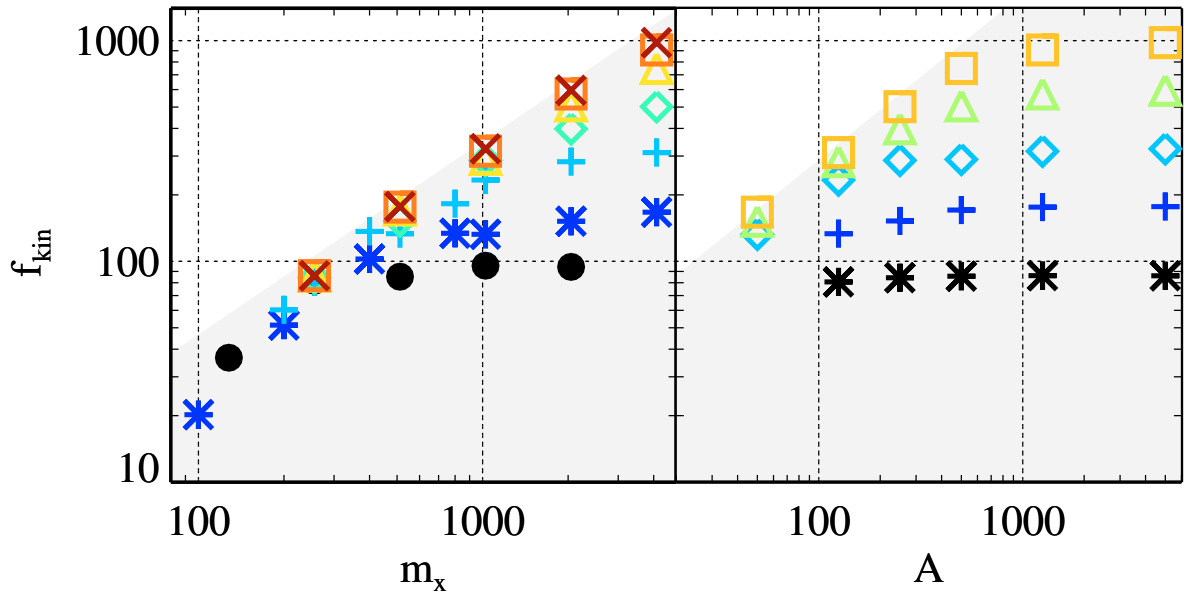

Fig. 8. Amplification of the magnetic field during the kinematic amplification phase: the amplification factor, $f_{\text {kin }}$ (see Eq. (14)), is shown for the models with an initial Mach number $\mathrm{M}=1$ as a function of the number of grid zones, $m_{x}$ (left panel), and of the initial Alfvén number, A (right panel). In the left panel, models with $A=25,50,125,250,500,1250$, and 5000 correspond to black circles, dark blue asterisks, light blue plus signs, green diamonds, yellow triangles, orange squares, and red $x$ signs, respectively. In the right panel, models with a grid size of $m_{x}=256,512,1024,2048$, and 4096 zones are displayed by black asterisks, dark blue + signs, light blue diamonds, green triangles, and orange squares, respectively. The upper border of the gray shaded regions is approximately given by the power laws $m_{x}^{7 / 8}$ and $\mathrm{A}^{3 / 4}$, respectively. given by $l_{b}$. In simulations, the decrease of $l_{b}$ can properly be followed only as long as $l_{b} \gtrsim \Delta_{\mathrm{g}}$, where $\Delta_{\mathrm{g}}$ is the finite grid spacing. When this limit is reached the exponential amplification of the field strength and field energy ceases. Further growth only regards the magnetic energy, which can increase at most linearly with time due to the increasing length of the sheet (at a constant width!). This point in the evolution marks the end of the phase of kinematic amplification.

Consequently, there exists an upper limit for the amplification of the magnetic field strength attainable by flux-sheet stretching that depends on the grid resolution. However, this limit set by the ratio of the grid spacing and the initial thickness of the flux sheet can only be reached, if the field strength remains dynamically negligible (i.e. below equipartition strength) during the kinematic amplification phase. This applies to models with weak initial magnetic fields $(A \gtrsim 1000)$, which are located near the upper border of the gray shaded region in the left panel of Fig. 8.

If the magnetic field reaches - within a factor of order unity - local equipartition strength during the kinematic amplification phase, the flow dynamics and as a consequence the termination of that phase show distinct features. This is the case for models with strong initial magnetic fields $(A \lesssim 500)$ and sufficiently fine resolution, which are located near the upper border of the gray shaded region in the right panel of Fig. 8. For these models $f_{\text {kin }} \propto A^{3 / 4}$, i.e., the amplification is larger for weaker initial fields. One factor contributing to this trend is the back-reaction of the field onto the flow. When locally the Alfvén number approaches the order of unity (see, e.g., the lower panel of Fig. 9), magnetic stresses start to decelerate the fluid in the flux sheets, and as the flux sheets partially thread the KH vortex its rotational velocity decreases, too. Consequently, the amplification factor will be smaller in this case than for an initially less strongly magnetized model. Finally, note that for models with weak initial magnetic fields $(A \gtrsim 1000)$ we do not observe effects due to back-reaction, as this requires larger field amplification factors than reached in our simulations due to insufficient grid resolution (see discussion above).

A second important issue for understanding our results is the effect of numerical resistivity. Although we integrate the equations of ideal MHD, the numerical scheme employed in our code mimics to some degree the effects of physical resistivity due to its inherent numerical resistivity. Thus, the numerical scheme smooths sharp features in the magnetic field and causes violent resistive instabilities of, e.g., tearing-mode type. The latter effect is most pronounced at length scales close to the grid spacing $\Delta_{\mathrm{g}}$.

When the typical length scales of the magnetic field - given approximately by $l_{b}$ - are comparable to the grid spacing $\Delta_{\mathrm{g}}$, we expect numerical resistivity to be important. For the model with $\mathrm{M}=1, \mathrm{~A}=125$, and $m_{x}=2048$ zones $l_{b} \approx \Delta_{\mathrm{g}}$ inside the flux sheet near the end of the kinematic amplification phase (Fig. 9, upper panel). The magnetic field is dominated by a complex pattern of sheets partially arranged in pairs or even triplets with anti-parallel fields. An example is the triple sheet structure passing roughly diagonally through the origin from down left to top right (Fig. 9, upper panel). This triplet consisting of a central sheet with $b^{x}>0$ and two parallel "wing" sheets with $b^{x}<0$ is the result of the advection of magnetic flux towards the central sheet by the flow.

As the advection continues the strength of the magnetic field in the side sheets increases, while their width decreases leading to intense currents. Eventually $l_{b} \leq \Delta_{\mathrm{g}}$, and resistive instabilities (tearing modes) start to grow, which curl up the two wing sheets and eventually disrupt them leaving behind only the central sheet (Fig. 9, upper panel). This process affects the entire triple sheet structure (Fig. 9, lower panel).

Shortly afterwards, the central sheet of the former triplet, still intact, is disrupted. From the interior of the vortex further sheets of magnetic flux are expelled creating new strong currents that again suffer strong resistive instabilities. This cycle of processes repeats every time strong currents build up by approaching flux sheets. As a consequence, the large coherent flux sheet structures are disrupted, and reconnection of magnetic field lines leads to numerous small-scale field structures including closed field loops, similarly to those reported in previous simulations (e.g., Keppens et al. 1999).

The amplification of the magnetic field terminates due to the development of these resistive instabilities, because (i) they convert magnetic energy into thermal energy; and because (ii) the resulting small-scale field and flow is less efficient in amplifying the magnetic field than a more coherent flow.

The mechanism just described is responsible for the termination of the kinematic amplification phase in well resolved models. All models with $\mathrm{A}=50, m_{x}>256$ and $\mathrm{A}=125, m_{x}>1024$ undergo this evolution. For even finer grids the results are essentially converged in terms of the amplification factor $f_{\text {kin }}$ (Fig. 8). Finding convergence for a flow whose behavior depends strongly on numerical resistivity is a remarkable result that deserves some 
explanation. Naturally, one would expect that with finer grid resolution (i.e., decreasing numerical resistivity) tearing modes are better suppressed, thus enabling the field to grow stronger.

However, this reasoning does only apply, if the main effect of numerical resistivity is the disruption of isolated flux sheets. In such a situation, the magnetic field in the flux sheet will be amplified until tearing modes grow faster than the field strength increases. As soon as the stretching of the flux sheet leads to a combination of a sufficiently strong field and a sufficiently thin sheet (both conditions as well as an increasing resistivity imply higher growth rates of resistive instabilities; see e.g., Biskamp 2000) tearing modes would start to disrupt the sheet. The amount of stretching necessary to reach this state depends on the resistivity, i.e., in our case on the grid resolution: finer grids require stronger fields and thinner sheets for disruption. Hence, the maximum field strength achievable at disruption should grow with increasing grid resolution, but the situation in our models described above is crucially different. Instead of operating on an isolated current sheet in a static background, the resistive instabilities terminating the growth of the magnetic energy act in our models on a multitude of flux sheets approaching each other closely due to a dynamic background flow. Their growth rates can become faster than the kinematic amplification of the field once the distance, $D_{\mathrm{s}}$, between two sheets rather than the width of the sheets, $l_{b}$, becomes sufficiently small, i.e, $D_{\mathrm{s}} \lesssim \Delta_{\mathrm{g}}$, but $l_{b}>\Delta_{\mathrm{g}}$. Contrary to the sheet width $l_{b}$, the distance $D_{\mathrm{s}}$ is not related to the magnetic energy stored in the sheets, but it is determined mainly by the flow field. Hence, there exists a relation between the velocity field and the instance of growth termination. The velocity field, in turn, depends mainly on the hydrodynamics of the $\mathrm{KH}$ vortex, and only weakly on the grid resolution, i.e., the moment when the flux sheets break up is independent of resolution. The latter also holds for the energy contained in the sheets. Converged results for the amplification factor can therefore be obtained despite the presence of a grid spacing dependent numerical resistivity.

As we saw above, the tearing modes of our models are triggered first after the formation of multiple sheet structures. At this point the central flux sheet of the triplet passing through the origin is still well resolved by several zones $\left(l_{b} \approx\right.$ a few $\left.\times \Delta_{\mathrm{g}}\right)$, but the distance $D_{\mathrm{s}}$ between the side sheets and the central sheet approaches $\Delta_{\mathrm{g}}$ as the former are advected towards the latter one.

Some of our model sequences show no convergence behavior (Fig. 8, left panel), as the grid resolution necessary for that increases with the initial Alfvén number. For very weak initial fields $(A \geq 250)$ even our finest grid with $4096^{2}$ zones does not yield a resolution-independent amplification factor. However, as the advection of the flux sheets does not depend on resolution and only weakly on the strength of the initial field (except for the sheets feedback is very limited in the kinematic amplification phase), the formation of unstable multiple sheets is possible even on coarse grids. Nevertheless, we do not observe strong resistive instabilities during this phase for these models.

We showed above that the growth rate of resistive instabilities during the kinematic amplification phase depends, apart from the resistivity, on the width of the flux sheet and the field strength, and that this phase ends when the tearing modes grow faster than the field is kinematically amplified by the velocity field. To match this condition, sufficiently strong fields are required during close encounters of flux sheets. This fact explains why we do not find resistive instabilities in models with too weak initial fields or too coarse resolution. In these cases the limitation of the maximum field strength of a flux sheet imposed by its minimum (resolvable) width leads to a reduced growth rate of resistive instabilities even when $D_{\mathrm{s}} \approx \Delta_{\mathrm{g}}$, i.e., the distance between two flux sheets is reduced to the grid spacing. Thus, these instabilities cannot terminate the kinematic field amplification process the same way as they do it in the case of stronger initial fields or finer grids.

The field strength required for resistive instabilities to terminate the kinematic amplification phase depends on the flow field: faster shear flows require stronger fields. Empirically, we find that the maximum field strength at termination corresponds roughly to an Alfvén number of order unity, i.e., to field strengths similar to those required for dynamic feedback.

To summarize, we find that there exist two different mechanisms to terminate the kinematic amplification phase.

- Passive termination: the magnetic field strength reaches a maximum when the decreasing thickness of the flux sheets approaches the grid spacing, i.e., when $l_{b} \approx \Delta_{\mathrm{g}}$.

- Resisto-dynamic termination: the magnetic field reaches equipartition strength with the flow field when a combination of dynamic and resistive processes terminate further field growth. Lorentz forces reduce the rotational velocity of the $\mathrm{KH}$ vortex, while resistive instabilities develop as flux sheets merge.

Whereas passive termination is a numerical artifact due to finite grid resolution, resisto-dynamic termination can be expected to occur in nature. The latter process leads to Alfvén velocities that are locally comparable with the shear velocity, and it is rather independent of the initial field strength. The volume average of the magnetic energy, on the other hand, increases with increasing initial field strength, because the volume filling factor of the magnetic field increases with the initial field strength.

Total amplification: The total amplification of the magnetic field is given by its growth during both the $\mathrm{KH}$ and the kinematic amplification phases.

According to our results the field amplification factor $f_{\text {kin }}$ (Eq. (14)) scales with the initial Alfvén number, A, approximately as $\mathrm{A}^{3 / 4}$ (see Fig. 8). Consequently, the maximum Maxwell stress obtainable at the end of the kinematic amplification phase scales with the initial magnetic field $b_{0} \propto \mathrm{A}^{-1}$ approximately as

$\mathrm{M}_{x y}^{\max } \propto b_{0}^{5 / 4}$

since $\mathrm{M}^{\max }=f_{\text {kin }} \mathrm{M}_{x y}^{\mathrm{KH}}$ (see Eq. (14)), and $\mathrm{M}_{x y}^{\mathrm{KH}} \propto b_{0}^{2}$ (i.e., the growth of the Maxwell stress during the $\mathrm{KH}$ growth phase is practically independent of the field). Note that this maximum value is only reached for a sufficiently fine grid resolution. If the model is under-resolved, $\mathrm{M}_{x y}^{\max }$ is reduced by a factor approximately $\propto m_{x}^{7 / 8}$, i.e., the maximum obtainable magnetic field strength depends on the strength of the initial magnetic field. Furthermore, as weak initial fields imply weak termination fields, which modify the dynamics of the flow only weakly, there exists a hydrodynamic limit of the magnetic KH instability.

The total amplification factors for the magnetic energy, $f^{e}$, and the magnetic field strength, $f^{b}$, are listed for various models in Table A.3 and displayed in Fig. 10. The trends described above also hold here. The amplification factors increase with finer grid resolution and eventually converge, the resolution required for convergence being higher for weaker fields. The converged amplification factors are larger for weaker magnetic fields, scaling as $f^{e} \propto b_{0}^{-2 / 3}$ and $f^{b} \propto b_{0}^{-1}$, respectively. Note that the latter scaling implies a maximum field strength that is independent of the initial field strength, consistent with the fact that 

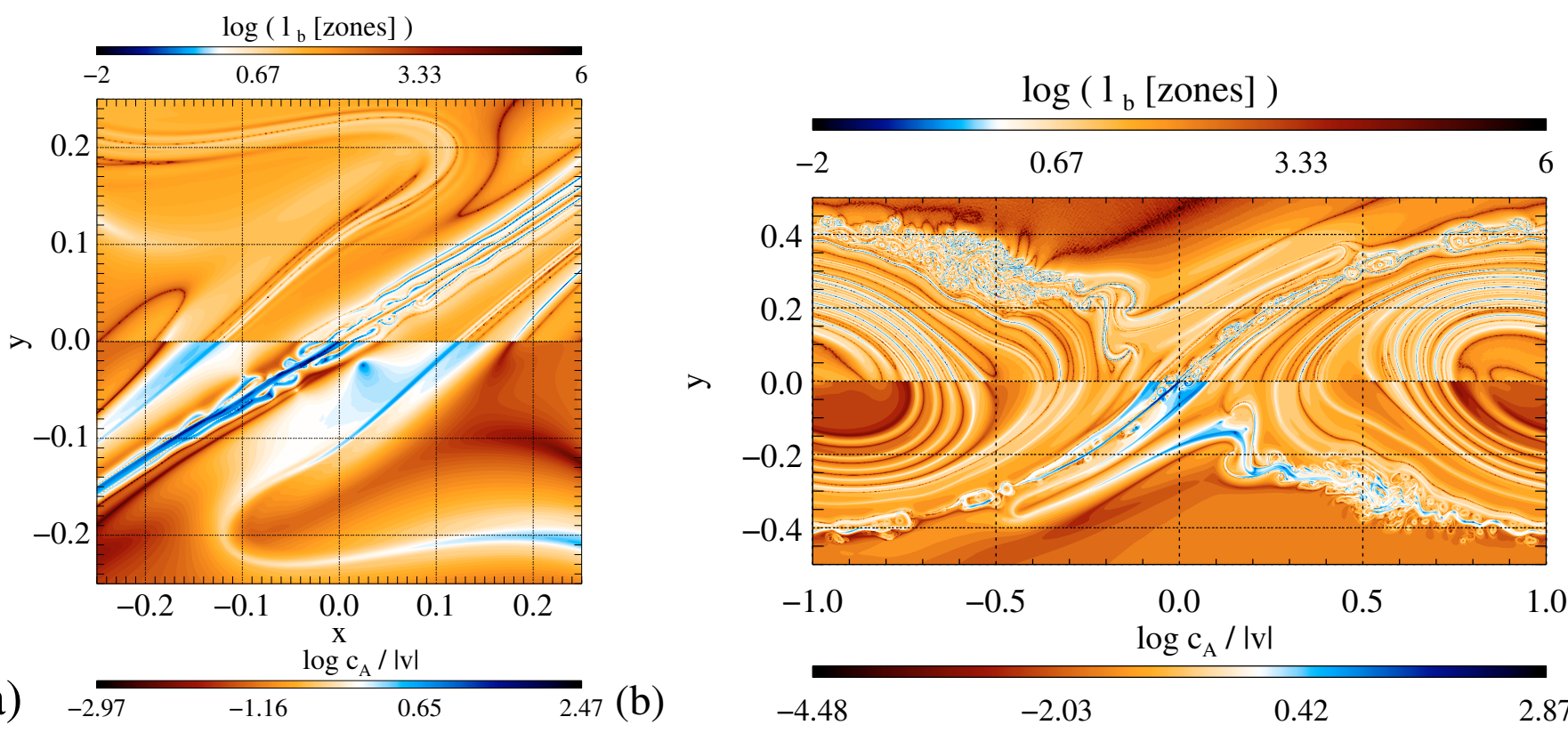

Fig. 9. Snapshots of the structure of the model with an initial Mach number $M=1$ and an Alfvén number $A=125$ taken close to the termination of the kinematic amplification phase (panel a)), and shortly afterwards (panel b)). The top half of each panel shows the logarithm of the characteristic length scale of the magnetic field, $|\boldsymbol{b}| /|\boldsymbol{\nabla} \times \boldsymbol{b}|$ in units of the zone size; reddish colors indicate regions where magnetic structures are larger than one computational zone, and bluish colors where they are smaller. The bottom half of each panel shows the logarithm of the ratio of the Alfvén velocity and the modulus of the fluid velocity, bluish and reddish colors denoting strongly and weakly magnetized regions, respectively.

there exists a hydrodynamic limit of the magnetic $\mathrm{KH}$ instability for weak fields (see above).

For models differing by their initial hydrodynamic state (i.e, initial Mach number $\mathrm{M}$, and initial shear layer width $a$; see Sect. 4) both amplification factors scale very similarly with the initial field strength (Fig. 10). In models with a smaller initial Mach number but the same initial shear layer width $(\mathrm{M}=0.5$, $a=0.05$; filled green circles), and with the same initial Mach number but an initially wider shear layer $(\mathrm{M}=1, a=0.15$; red diamonds) the $\mathrm{KH}$ instability grows slower than in standard model $(\mathrm{M}=1, a=0.05)$ discussed above. It also saturates at smaller transverse kinetic energies $\left(\approx 3.3 \times 10^{-3}\right.$ and $\approx 4.2 \times 10^{-3}$, respectively, instead of $\approx 9.5 \times 10^{-3}$, which implies a slower kinematic amplification of the field. Hence, $f^{b}$ is smaller, but its scaling $\propto b_{0}^{-1}$ is similar to that of the reference models. Independent of the properties of the initial shear flow, we find $f^{e} \propto b_{0}^{-2 / 3}$, the proportionality constant depending, however, in a complex way on the initial state. For fixed shear layer width, slower shear flows lead to less efficient field amplification. The amplification factor of the magnetic energy $f^{e}$, on the other hand, is practically independent of the shear layer width, while $f^{b}$ decreases for narrower initial shear layers. However, since the volume where amplification takes place is larger than that given by the initial shear layer width, overall the total magnetic energy grows as in the case of a narrower transition layer.

To summarize, the maximum magnetic field achieved is mainly a function of the overturning velocity of the $\mathrm{KH}$ vortex, corresponding to the transverse kinetic energy, while the magnetic energy at the termination of the growth depends on the initial Mach number, on the width of the shear profile and on the initial magnetic field.

Saturation, dissipation and disruption: After termination of the amplification of the magnetic field, the shear flow enters the saturation phase. We will discuss in the following mainly models encountering a resisto-dynamic termination rather than a passive one, but also briefly mention the behavior of models suffering a passive termination of the field growth.

As a typical example, we illustrate the evolution of the partial energies of the model with $M=1$ and $A=125$ in Fig. 11 . After the end of the kinematic amplification phases both the kinetic energy $\propto v_{x}^{2}$ (shear component) and $\propto v_{y}^{2}$ (transverse component) decrease, while the internal energy increases. The magnetic energy remains roughly at the level it has reached at the end of the kinematic amplification phase. In the final state, the transverse kinetic energy is less than the total magnetic energy, and equal to the transverse magnetic energy.

To understand these results, we compare the model structure at the beginning of the saturation phase with that near the end of the simulation. According to Fig. 12 the model exhibits clear signs of disruptive dynamics (see Jones et al. 1997). The $\mathrm{KH}$ vortex is still visible as a coherent pattern at $t=34.4$, i.e., shortly after the end of the kinematic amplification phase (panel a). At $t=81.5$ the vortex is disrupted, the flow field is dominated by a broad transition region separating oppositely directed shear flows, and the $y$-component of the velocity shows small-scale structures (see patchy colors in upper part of Fig. 12, panel b). The magnetic field is concentrated into a multitude of thin flux sheets with a typical length scale $l_{b} \approx \Delta_{\mathrm{g}}$. Due to magnetic reconnection the sheets possess a complex topology. Several closed field loops have formed that are stabilized by a combination magnetic loop tension and total pressure $\left(P+b^{2} / 2\right)$. The flux sheet pattern is imprinted onto the flow field and the gas pressure distribution. Although the gas pressure is reduced inside the sheets there is sufficient magnetic pressure to keep the flux sheets in pressure equilibrium with their surroundings. That explains why the distribution of the total pressure is rather featureless. 

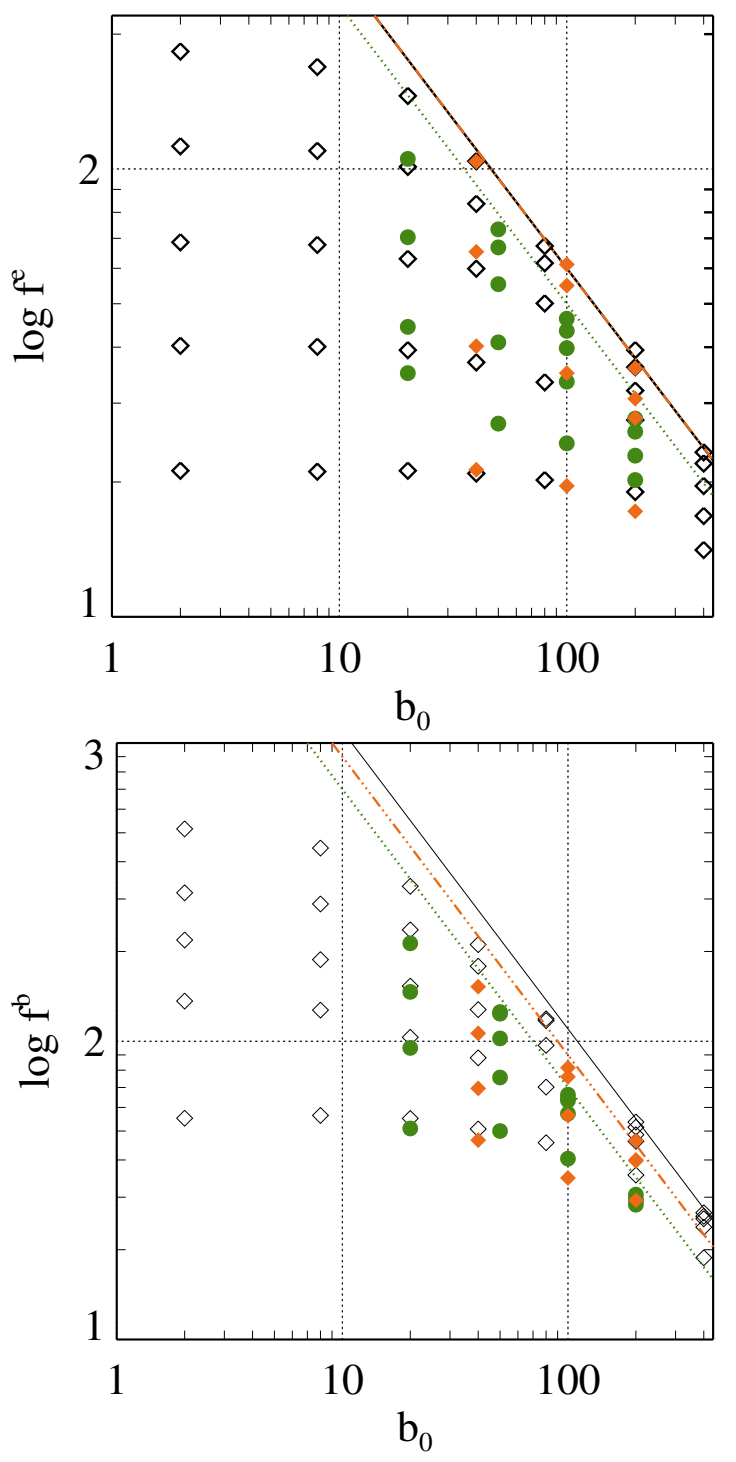

Fig. 10. The total amplification factors for the magnetic energy $f^{e}$ (top panel) and the magnetic field strength, $f^{b}$ (bottom panel) as a function of the initial magnetic field, $b_{0}$, for models with different initial shear flows: empty black diamonds, filled green circles, and filled red diamonds correspond to models with $\mathrm{M}=1$ and $a=0.05, \mathrm{M}=0.5$ and $a=0.05$, and $\mathrm{M}=1$ and $a=0.15$, respectively. The spread in vertical direction reflects different grid resolutions. To indicate the scaling of the amplification factors with the initial field strength, the figure also gives power laws $\propto b_{0}^{-2 / 3}$ (top panel), and $\propto b_{0}^{-1}$ (bottom panel).

As visible in Fig. 9 (panel b) and Fig. 12 (panel a), the resistive instabilities responsible for the termination of the kinematic amplification phase spread along the flux sheets leading to a complex field topology and inhibiting further growth of the field not only locally but in the entire volume.

Locally, i.e. inside the flux sheets, the magnetic field is in equipartition with the velocity field (globally it is still an order of magnitude weaker). In resistive instabilities magnetic energy is converted into internal one. Since the magnetic field has been built up previously at the expense of the kinetic energy, the instabilities actually mediate the transformation of kinetic energy into internal energy, hence acting akin to a hydrodynamic viscosity. Eventually, a steady state (in a statistical sense) develops where the magnetic energy, and thus the effective viscosity, becomes a)

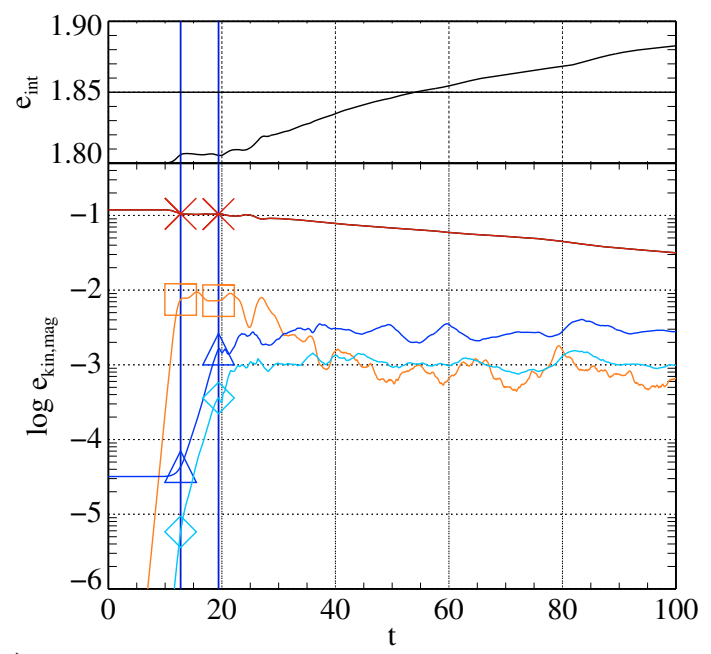

b)

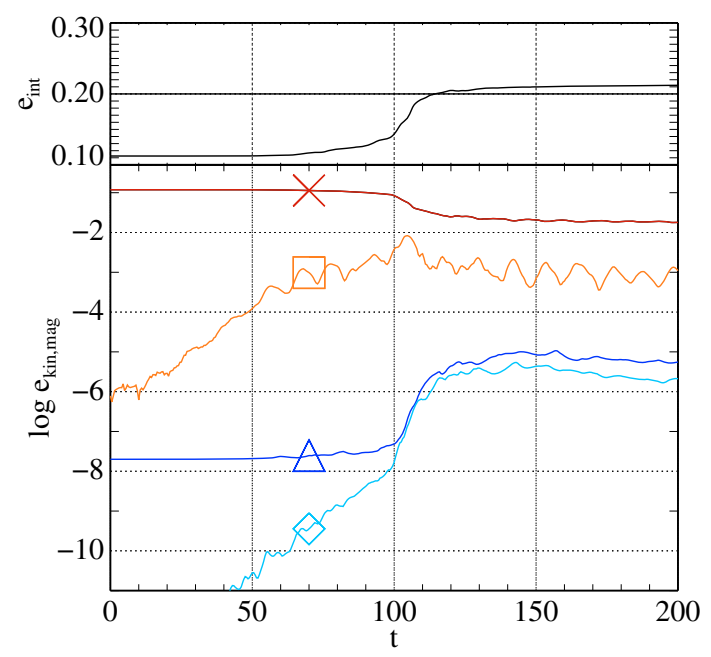

Fig. 11. Panel a): Evolution of the model with $M=1$ and $A=125$, computed on a grid of $2048^{2}$ zones. The top panel shows the internal energy density as a function of time. The bottom panel shows the logarithms of the volume-averaged kinetic energy densities $e_{\text {kin }}^{x}$ (dark red line, $\times$ signs) and $e_{\text {kin }}^{y}$ (orange line, squares), and of the volume-averaged magnetic energy densities $e_{\mathrm{mag}}^{x}$ (dark blue line, triangles) and $e_{\mathrm{mag}}^{y}$ (light blue line, diamonds), respectively. The two vertical lines indicate the end of the $\mathrm{KH}$ growth (left) and kinematic amplification (right) phase, respectively.

Panel b): Same as panel a), but for a supersonic model with $M=4.4$ and $A=5000$. Because of the model's completely different dynamics, the lines indicating the end of the growth phases are omitted.

time-independent, while kinetic energy is converted into internal one at a constant rate. After the disruption of the $\mathrm{KH}$ vortex, the transverse velocity reflects the turbulence resulting from the resistive instabilities, i.e., $e_{\text {kin }}^{y}$ is a measure (like the magnetic field strength) of the intensity of turbulence. Consequently, $e_{\mathrm{kin}}^{y}$ remains constant at saturation, and the disruption of the $\mathrm{KH}$ vortex can be identified by the instance when $e_{\text {kin }}^{y} \approx e_{\text {mag }}^{y}$.

The saturation level of the magnetic field, and thus the effective viscosity, is set by its level at the termination of the kinematic amplification phase. This level decreases with decreasing initial field strength, i.e. the weaker the initial field the slower is the resistive disruption of the KH vortex. To quantify this effect, we define a disruption time, $t_{\mathrm{dis}}$, as the time when $e_{\mathrm{kin}}^{y}$ falls below 
M. Obergaulinger et al.: Local simulations of the magnetized Kelvin-Helmholtz instabilityin neutron-star mergers

(a)
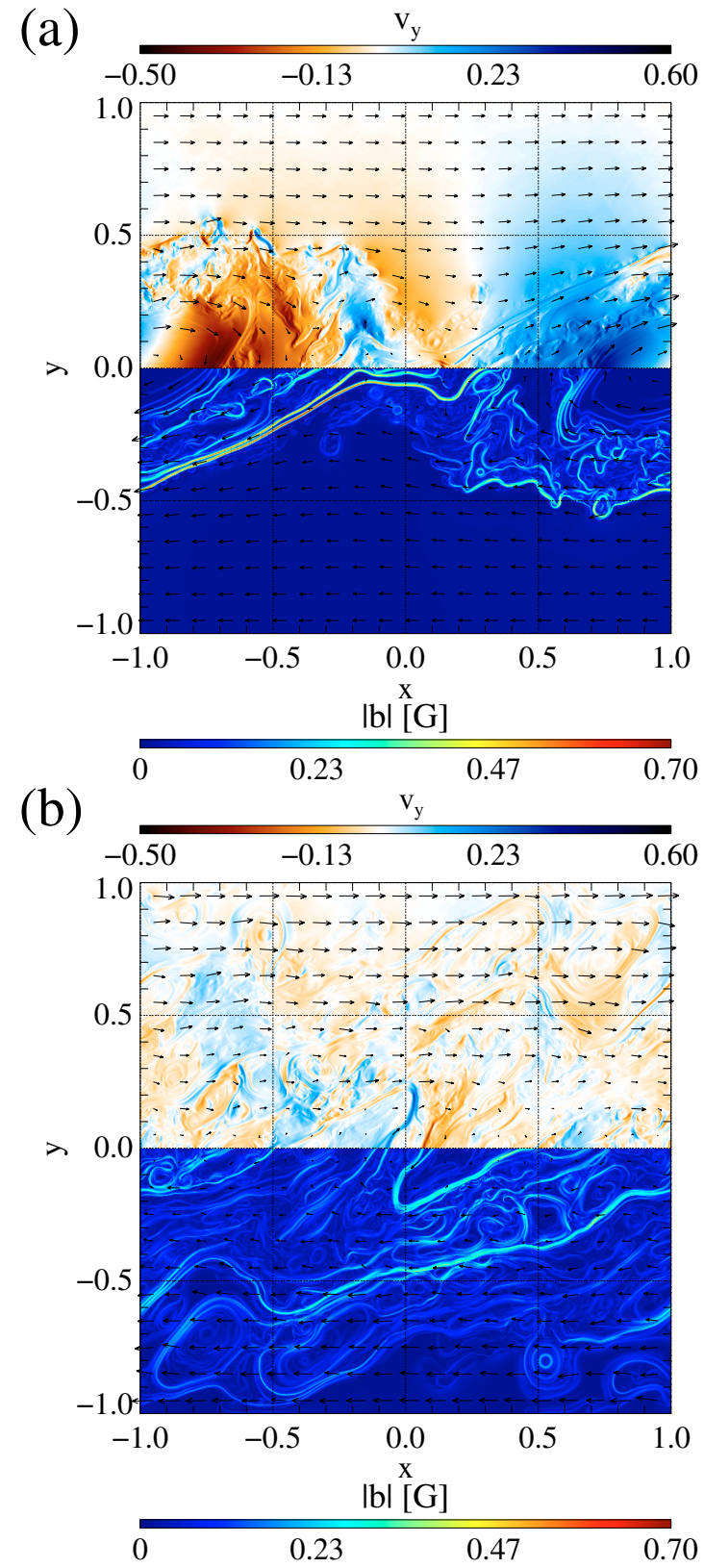

Fig. 12. Structure of the model with $M=1$ and $A=125$ computed on a grid of $2048^{2}$ zones near the beginning of the saturation phase at $t=34.4$ (upper panel), and at $t=81.5$ (lower panel), respectively. The top and bottom half of each panel shows the $y$-component of the velocity and the modulus of the magnetic field, respectively. The flow field is illustrated by the black arrows.

$e_{\text {mag }}^{y}$, and a deceleration rate $\sigma_{\mathrm{dec}} \equiv \partial_{t} \log E_{\mathrm{kin}}^{x}=1 / t_{\mathrm{dec}}{ }^{2}$. Both quantities are listed in Table A.4, and $t_{\mathrm{dis}}$ and $t_{\mathrm{dec}}$ are also shown as a function of the initial Alfvén number in Fig. 13. For models with very weak deceleration, the evolution of the kinetic energy is dominated by large oscillations. Thus, the determination of the value of $\sigma_{\text {dec }}$ is uncertain to some degree in these cases, and the numerical values quoted in Table A. 4 should be taken with care.

Depending on the initial field strength, the models require a certain minimum resolution to obtain converged values for $t_{\mathrm{dis}}$ and $t_{\mathrm{dec}}$, respectively. If the resolution is too low, the disruption

\footnotetext{
${ }^{2}$ We also considered alternative definitions of $\sigma_{\text {dec }}$ that, however, do not change the arguments in the discussion below.
}

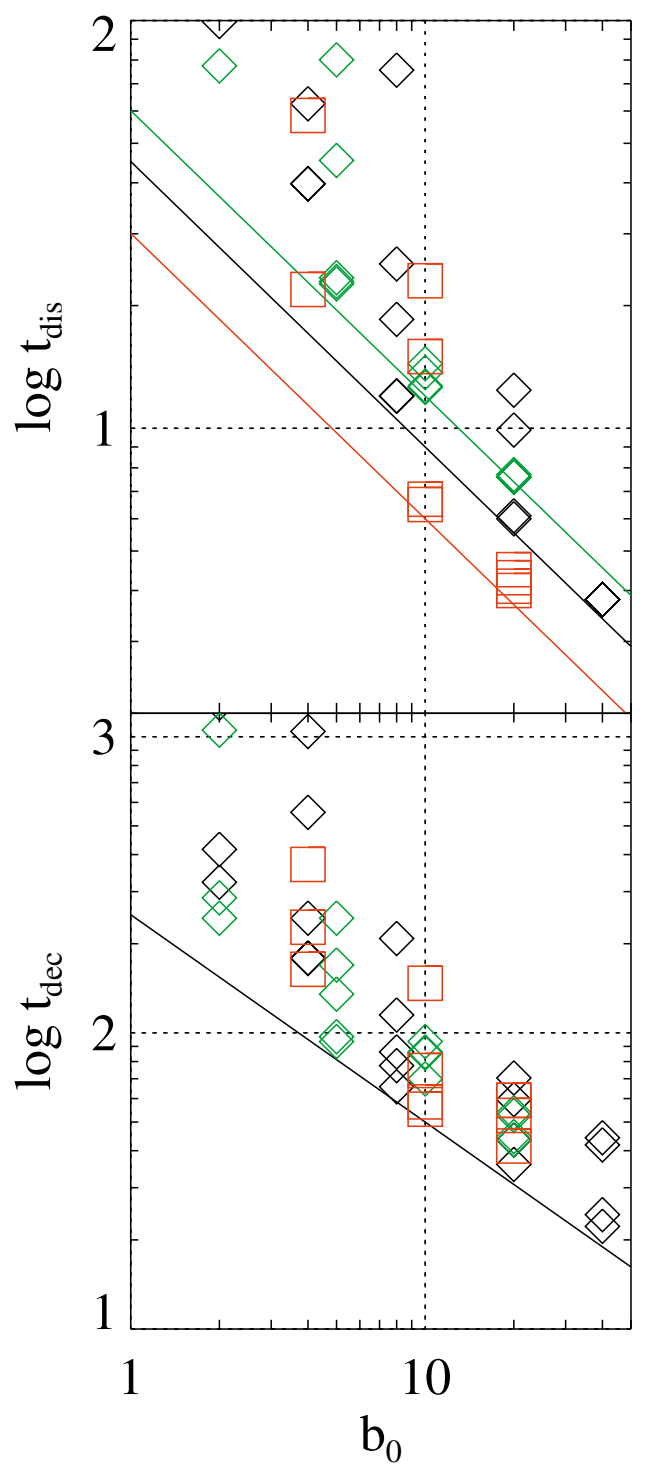

Fig. 13. Time scales for the disruption of the KH vortex $t_{\text {dis }}$ (upper panel) and deceleration of the flow, $t_{\mathrm{dec}}$ (lower panel) as a function of the initial field strength $b_{0}$. The various models are represented by different symbols: black diamonds, green diamonds, and red squares correspond to models with $\mathrm{M}=1$ and $a=0.05, \mathrm{M}=0.5$ and $a=0.05$, and $\mathrm{M}=1$ and $a=0.15$, respectively. The vertical spread of identical symbols reflects different grid resolutions finer resolution yielding smaller values of $t_{\mathrm{dis}}$ and $t_{\mathrm{dec}}$, respectively. The lines $\propto b_{0}^{-0.7}$ indicate the approximate scaling of the time scales with $b_{0}$.

of the vortex and the deceleration of the shear flow proceed too slow due to an insufficient amount of field amplification. In the following, we will focus on converged or nearly converged models.

The disruption and deceleration time scale with the initial field strength roughly as $b_{0}^{-0.7}$. Comparing these times for models with different initial shear profiles, we find that the disruption time depends sensitively on both $\mathrm{M}$ and the initial shear layer width $a$. The larger the amplification factor of the magnetic en$\operatorname{ergy} f^{e}$ is for a given shear profile (see Fig. 10), the faster is the disruption of the vortex. The deceleration time, on the other hand, shows a weaker dependence on $\mathrm{M}$ and $a$. Even for $a=0.2$, which implies a much slower KH growth and a very low saturation level of $e_{\text {kin }}^{y} \sim 10^{-3}$, the deceleration time is very similar to 
that of the models discussed above, although the magnetic field strength is much smaller.

For weaker fields, whose growth ends due to passive instead of resisto-dynamic termination (i.e., non-converged models), the kinetic energy decreases much more slowly. Resistive instabilities grow much slower in such models, because of the growth of their field strength is restricted by numerical resolution. Hence, the effective viscosity is much lower in these models than in well resolved ones.

\subsection{Supersonic shear flows}

We simulated supersonic shear flows with a Mach number $M=4.4$ using the same velocity profile as for the model with $\mathrm{M}=1$, but a reduced gas pressure of $P=0.0375$. In the following, we compare models with very large $(A=5000)$ and small $(A=25,50)$ Alfvén numbers. For the simulations we used grids with a resolution between $128^{2}$ and $2048^{2}$ zones. As the main result we find that the growth rate of the magnetic field is lower in supersonic shear flows than in sonic and subsonic shear flows.

For $A=5000$, none of the simulations shows an effect of the magnetic field on the flow. For all grid resolutions the early evolution of the magnetic model (shock formation and interaction) is similar to that of the non-magnetic one. Until $t \sim 70$ the transverse kinetic energy increases roughly exponentially before leveling off (Fig. 11, panel b). The magnetic field in $y$-direction is amplified at a similar rate as the kinetic energy until $t \approx 100$, when the amplification rate increases strongly. This phase of efficient field growth, lasting until $t \approx 130$, corresponds to the formation of large regions of subsonic flow where most of the field amplification occurs. The magnetic field is concentrated in thin sheets. While dominated by a multitude of shock waves during early phases, the model shows a subsonic vortical flow in the final state, similarly to the models discussed in the previous subsection. The kinetic energy has decreased by a factor of four during the entire evolution. Most of this deceleration has occurred during the early saturation phase of the $\mathrm{KH}$ instability when the magnetic field is amplified most strongly.

Comparing the evolution of the magnetic energy for simulations with different grid resolution, we find trends similar to those of subsonic models with dynamically negligible fields. Stronger magnetic fields are obtained for finer grids the explanation for this behavior being the same as that for the resistodynamic termination for subsonic shear flows: amplification ceases when the width of a flux sheet becomes comparable to the grid spacing.

In models with $A=25$ and $A=50$ the magnetic field modifes the dynamics. In early stages, a number of weak shock waves form. Interacting with magnetic flux sheets close to the shearing interface, theses shocks are disrupted. Spreading away from the interface in positive and negative $y$-direction, a wide region of subsonic flow forms. Both its geometry and formation differ from those of subsonic shear flows. In barely magnetized models, a subsonic flow possessing a considerable transversal extent results from the interaction of oblique shocks (see Sect. 4.2), whereas in more strongly magnetized models the magnetic field enforces a subsonic region elongated along the $x$-direction. We find convergence with respect to the saturation level of the magnetic energy, whose value is in general below the value of subsonic models. At late times, we observe equipartition between the transverse kinetic energy and the magnetic energy. The deceleration times of the flow are fairly similar to those of the non-magnetic models.

\subsection{Anti-parallel initial fields}

We have recomputed a number of models with anti-parallel initial fields, i.e., an initial field $b^{x}=b_{0}^{x} \operatorname{sign}(y)$. Similar simulations were performed previously by Keppens et al. (1999), whose results we confirm.

For strong initial fields, corresponding to an initial Alfvén number $A=5$, we observe in accordance with Keppens et al. (1999) a destabilization of the shear layer with respect to the non-magnetic case.

The qualitative dynamics of initially weakly magnetized shear flows with is anti-parallel fields is similar to the case of parallel initial fields, evolving through the three phases described in Sect. 4.3. There are, however, quantitative differences concerning, e.g., the saturation value of the magnetic energy or the deceleration rate. The KH growth phase is similar for both field configurations, as is the growth rate of the magnetic field during the kinematic amplification phase. The termination of the latter phase depends, however, on the initial field orientation: for the same initial Alfvén number, a model with anti-parallel initial field experiences less amplification than one with parallel magnetic fields. The modes of termination of the kinematic amplification phase are the same as in the case of parallel fields (passive or resisto-dynamic termination), but due to the presence of oppositely directed flux sheets right from the beginning of the evolution reconnection of field lines is enhanced. This leads to earlier termination, i.e.,lower termination field strengths. As a consequence, the magnetic deceleration of the $\mathrm{KH}$ vortex is less efficient in case of initially anti-parallel field. The disruption times and deceleration timescales are a factor of $\sim 2 . .3$ larger than those measured for parallel-field models.

\section{Three-dimensional models}

In the following section, we study the evolution of $\mathrm{KH}$ instabilities in three-dimensional shear flows. Obviously, the numerical resolution we can afford in $3 \mathrm{D}$ is much worse than in our best resolved 2D models. This prevented us from performing a study as detailed as in the two-dimensional case. The $3 \mathrm{D}$ models we have simulated are listed in Table A.5.

\subsection{Subsonic shear flows, parallel magnetic field}

\subsubsection{Non-magnetic models}

In $3 \mathrm{D}$ the $\mathrm{KH}$ vortex is unstable against (purely) hydrodynamic instabilities Ryu et al. (2000): coherent vortex tubes near the main $\mathrm{KH}$ vortex exert non-axial stresses on the vortex, and fluid elements are prone to the so-called elliptic instability, an instability caused by time-dependent shear forces, which act on fluid elements while they orbit the vortex on elliptic trajectories. The result is isotropic decaying turbulence.

As in $2 \mathrm{D}$, we seeded the $\mathrm{KH}$ instability with small perturbations of the $y$-component of the velocity varying sinusoidally in $x$-direction (see Eq. (9)). To break the translational symmetry in $z$-direction, we added a small random perturbation $v_{\text {rndm }}$ to all velocity components, where

$v_{\text {rndm }}=\xi_{\text {rnd }} v_{y}^{0} \quad$ with $\quad \xi_{\text {rnd }} \in\left[10^{-4}, 1\right]$.

In the non-magnetized reference model a KH vortex tube elongated in $z$-direction forms during the exponential growth of the instability. The vortex tube is clearly visible in the (front part of the) lower panel of Fig. 14, which shows the vorticity distribution at $t=10$ (shortly after the termination of the growth of the instability), and at $t=50$ (in the non-linear phase), respectively. 
M. Obergaulinger et al.: Local simulations of the magnetized Kelvin-Helmholtz instabilityin neutron-star mergers

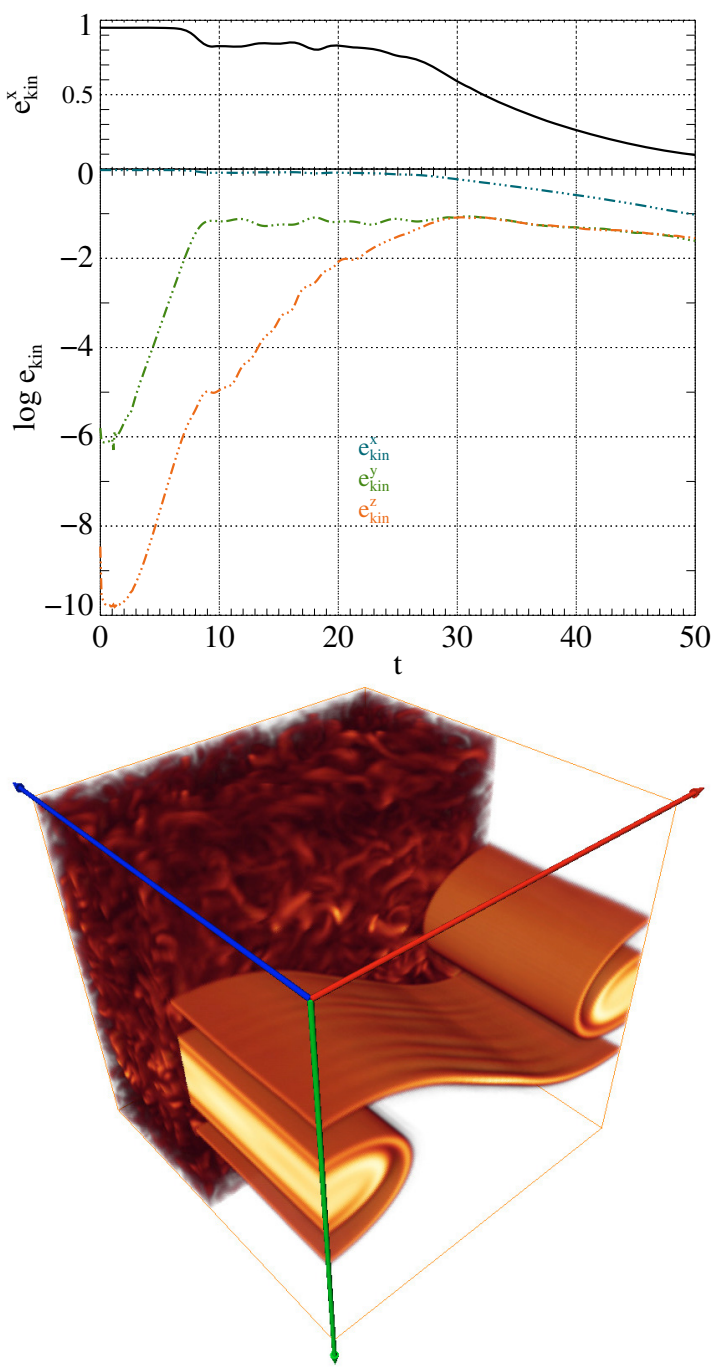

Fig. 14. Upper panel: temporal evolution of the volume-averaged kinetic energy densities defined in Eq. (10) for a 3D non-magnetized model. The upper part shows $e_{\text {kin }}^{x}$, and the lower one the logarithm of $e_{\mathrm{kin}}^{x}$ (blue), $e_{\mathrm{kin}}^{y}$ (green), and $e_{\mathrm{kin}}^{z}$ (red lines), respectively. Lower panel: volume rendering of the modulus of the vorticity, $|\boldsymbol{\nabla} \times \boldsymbol{v}|$, of the same simulation at two different times. The computational box (red, green, and blue arrows point into $x, y$, and $z$-direction, respectively) is divided into two halves: the front half shows $|\nabla \times \boldsymbol{v}|$ at $t=10$ when the KH vortex tube is still fully intact, and the back half at $t=50$ after the complete disruption of the vortex tube by secondary instabilities.

The temporal behavior of the volume-averaged kinetic energy densities defined in Eq. (10) reflects the evolution of the flow (Fig. 14). Up to $t \approx 7, e_{\text {kin }}^{x}$ is practically constant. Then it starts to drop by about $20 \%$ within two time units when the forming vortex tube extracts kinetic energy from the shear flow. Afterwards $e_{\text {kin }}^{x}$ stays again approximately constant until the elliptic instability begins to destroy the vortex tube at $t \approx 20$. The transverse kinetic energy densities, $e_{\text {kin }}^{y}$ and $e_{\text {kin }}^{z}$, show exponential growth before saturating at the same level. Note that $e_{\text {kin }}^{z}$ saturates about 20 time units later than $e_{\text {kin }}^{y}($ at $t \approx 30)$, because it starts growing from an initial value that is a factor of $10^{4}$ smaller. In addition, its growth rate, which is similar to that of the magnetic energy during the kinematic amplification phase of $2 \mathrm{D}$ models, decreases after the end of the $\mathrm{KH}$ phase $(t \approx 9)$ when the elliptic instability developing along the vortex tube takes over $(t \gtrsim 12)$. The latter saturates when the vortex tube

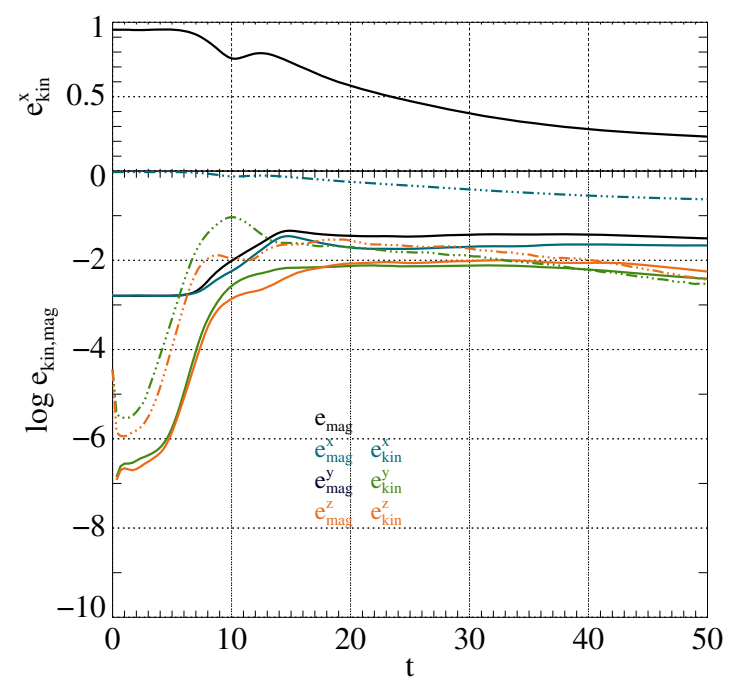

Fig. 15. Temporal evolution of various energy densities of a 3D KH model having an initial Mach and Alfvén number of $M=1$ and $A=50$, respectively. The amplitude of the imposed random perturbation was comparable to that of the sinusoidal one, i.e., $\xi_{\text {rnd }}=1$ (see Eq. (17)). The top panel shows the evolution of $e_{\mathrm{kin}}^{x}$. The bottom panel illustrates the evolution of the volume-averaged total magnetic energy density (black solid line), and of the magnetic energy densities corresponding to the three field components: $e_{\mathrm{mag}}^{x}$ (blue solid line), $e_{\mathrm{mag}}^{y}$ (green solid line), and $e_{\mathrm{mag}}^{z}$ (red solid line), respectively. The dash-triple-dotted lines show the corresponding kinetic energy densities $e_{\mathrm{kin}}^{x}, e_{\mathrm{kin}}^{y}$, and $e_{\mathrm{kin}}^{z}$ using the same color coding.

is disrupted, and $e_{\mathrm{kin}}^{z} \approx e_{\mathrm{kin}}^{y}$. Subsequently, turbulence develops (see vorticity pattern at $t=50$ in Fig. 14, lower panel), and the shear flow is strongly decelerated as indicated by the decrease of $e_{\text {kin }}^{x}$ (Fig. 14, upper part of upper panel). The deceleration is considerably faster than in the case of weakly magnetized 2D models.

\subsubsection{Weak-field models}

For weak-field models, the 3D KH vortex is subject to two different instabilities competing for its disruption: the purely hydrodynamic one discussed in the previous subsection, and the resistive ones analyzed in Sect. 4.3. Which of these instabilities is most efficient depends the importance of 3D effects, which in turn is determined by the initial amplitude of the random perturbations. Independently of the purely hydrodynamic instabilities, if there exists a (weak) magnetic field, it may also disrupt the vortex. In the latter case, the post-disruption flow shows a larger degree of organization than a non-magnetized one due to the prevalence of flux tubes and flux sheets where the magnetic and flow field are aligned.

For a model with $A=50$ and a strong random perturbation, i.e., comparable to the sinusoidal one $\left(\xi_{\text {rnd }} \approx 1\right.$; see Eq. (17)), the flow field shows considerable variations in $z$-direction already during the formation of the KH vortex tube (Fig. 15). During the kinematic amplification phase, we observe a pattern of thin vorticity tubes arising from magnetic flux tubes wound up around the dominant 3D vortex tube (located near the edge of the computational domain $x$-direction; see Fig. 16). The KH vortex tube is disrupted until the end of the kinematic amplification phase. At $t \approx 15$ the volume-averaged transverse kinetic energy densities $e_{\text {kin }}^{y}$ and $e_{\text {kin }}^{z}$ reach equipartition (see Fig. 15). Magnetic field amplification ceases at that point. The subsequent deceleration 


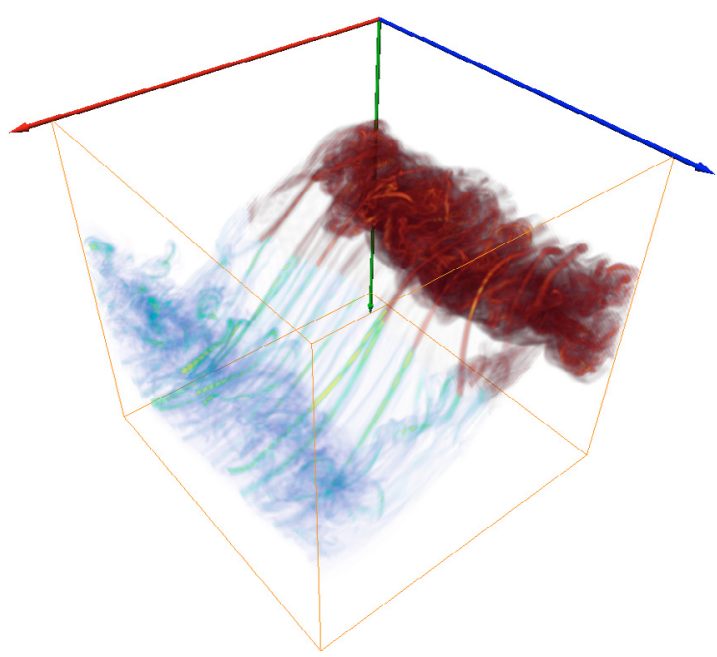

Fig. 16. Volume rendered magnetic field strength, $|\boldsymbol{b}|$ (blue-green) and modulus of the vorticity, $|\boldsymbol{\nabla} \times \boldsymbol{v}|$ (red-yellow) of a 3D KH model with initial Mach and Alfvén numbers $M=1$ and $A=50$, respectively. The snapshot is taken during the kinematic amplification phase $(t=9.21)$. The amplitude of the imposed random perturbation was comparable to that of the sinusoidal one, i.e., $\xi_{\text {rnd }}=1$ (see Eq. (17)). The computational domain is given by the thin red box. The red, green, and blue arrows indicate the $x, y$, and $z$ coordinate axes, respectively.

of the shear flow is mediated mainly by the hydrodynamic instabilities active also in non-magnetized models (see previous subsection). Hence, deceleration occurs with similar efficiency, but ceases when the transverse kinetic energy densities drop below the magnetic ones at $t \sim 50$ and the magnetic field begins to suppress the hydrodynamic instabilities. The final state of the model consists of decaying volume filling turbulence. Since deceleration is incomplete, the model retains a slower, smooth shear flow. The velocity and the magnetic field are dominated by their respective $x$-components, leading to considerably anisotropic turbulent fields.

Decreasing the amplitude of the random perturbation to $\xi_{\text {rnd }}=10^{-2}$ or even $\xi_{\text {rnd }}=10^{-4}$ (see Eq. (17)) while keeping the initial magnetic field fixed, the shear flows evolves very differently. For small random perturbations field amplification and overall dynamics proceed similarly as in 2D models during the $\mathrm{KH}$ growth and kinematic amplification phases regarding the formation of a flux sheet. Indeed, the $z$-variation of all physical quantities is very small, The dynamics of weak-field models is very similar to that of non-magnetized ones, too. During the $\mathrm{KH}$ growth phase, a vortex tube forms, which is oriented in $z$-direction.

As in 2D models the initial $\mathrm{KH}$ growth phase is followed by a kinematic amplification phase. This phase terminates, as in $2 \mathrm{D}$, depending on $\mathrm{A}$ and the grid resolution either passively or dynamically by the back-reaction onto the flow via Maxwell stresses and resistive instabilities. The kinematic amplification factor of the magnetic energy, $f^{b}$, is the same as in 2D.

For an initial Alfvén number $A=5000$ we find passive termination of the kinematic field amplification phase (Fig. 17). Since the magnetic field remains far too weak to affect the evolution, the dynamics resembles that of a non-magnetized model. Until $t \approx 30,3 \mathrm{D}$ hydrodynamic instabilities disrupt the $\mathrm{KH}$ vortex tube. Indicative for the development of these instabilities is the rise of $e_{\text {kin }}^{z}$ until it reaches equipartition with $e_{\text {kin }}^{y}$ at $t \approx 28$, growing at a rate comparable to the kinematic growth

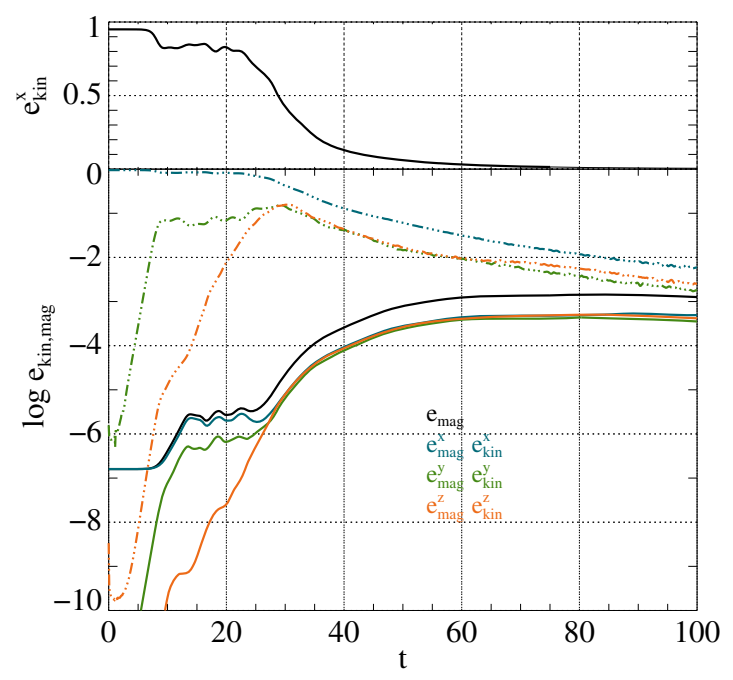

Fig. 17. Same as Fig. 15, but for a model with an initial Alfvén number $A=5000$.

rate of the magnetic field. The volume-averaged total magnetic energy density, and both $e_{\mathrm{mag}}^{x}$ and $e_{\mathrm{mag}}^{y}$ remain constant during this phase, only $e_{\text {mag }}^{z}$ increases exponentially. After termination of the 3D instabilities all volume-averaged magnetic energy densities are equal, growing slowly during the remaining evolution. Turbulence spreads across the entire computational volume and decelerates the shear flow with the same efficiency as in the nonmagnetized model.

For stronger initial fields (or finer grid resolution) the resistive instabilities terminating the kinematic amplification phase are accompanied by a rapid growth of the z-component of the velocity and the magnetic field. For models with $A=50$ and $\mathrm{A}=25$, this happens at $t \approx 15$. Despite this rapid growth, the influence of $3 \mathrm{D}$ effects remains moderate. At $t=15$ close to the end of the strong rise of $e_{\mathrm{mag}}^{z}$ and $e_{\mathrm{kin}}^{z}$, the topology of the velocity field and magnetic field is still dominated by a large planar structure resembling the flux sheet of 2D simulations.

This is a pronounced difference to the case of large random perturbations (compare Figs. 18 and 16). Note, however, that there is already some indication of the decay of the flux sheet into flux tubes in the small random perturbation case, too. After the dynamic-resistive termination of the kinematic amplification phase, the $z$-components of the magnetic field and the velocity start growing again although at a smaller rate, while the $x$ and $y$-components of the velocity are decelerated by the magnetic field. The decay of the flux sheet into tubes is almost complete at $t=25$ (right panel of Fig. 18) when $e_{\text {kin }}^{y} \approx e_{\text {kin }}^{z}$ and $e_{\text {mag }}^{y} \approx e_{\text {mag }}^{z}$ holds. In the subsequent saturation phase, turbulence develops, and the shear flow is decelerated at a rate similar to that of the 2D models.

Comparing the properties of the turbulence and the deceleration rate of $3 \mathrm{D}$ models with different initial field strength, different grid resolution, and different initial perturbations, we find that the intensity of the turbulent magnetic and velocity fields, and consequently the deceleration of the shear flow, is determined by the interplay of (3D) hydrodynamic instabilities, and (2D) magnetic stresses and instabilities:

Hydrodynamic disruption: if field amplification is too weak to prevent the dominance of hydrodynamic over hydromagnetic instabilities during the early evolution, the $\mathrm{KH}$ vortex tube is disrupted and the shear flow is decelerated at a rate similar to 


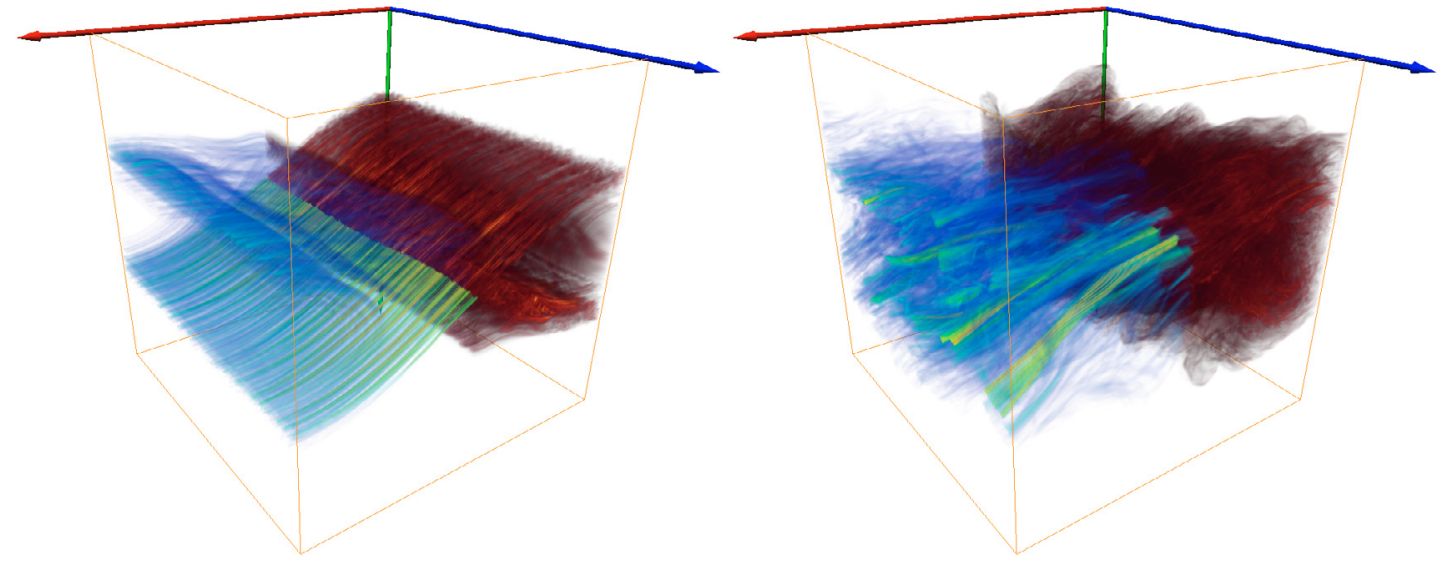

Fig. 18. Same as Fig. 16, but for a model where the amplitude of the imposed random perturbation was much smaller than that of the sinusoidal one, i.e., $\xi_{\text {rnd }} \ll 1$ (see Eq. (17)). The two snapshots are taken at $t=15$ (left) and $t=25$ (right), respectively.

that of the non-magnetic case. The magnetic field is amplified or sustained in the turbulent velocity field provided by the hydrodynamic instabilities. The evolution of this class of models tends towards isotropic decaying turbulence.

Hydromagnetic disruption: if the magnetic field leads to the disruption of the $\mathrm{KH}$ vortex tube before hydrodynamic instabilities can set in, the deceleration of the shear flow is driven by magnetic fields. In this case, the deceleration rate is similar to that of 2D flows, but it may also be smaller depending on the level of hydromagnetic turbulence, which is determined among other factors by the strength of the initial random perturbations, the grid resolution, etc. The turbulent final state of such models is dominated by a larger $x$-component of the magnetic field, the transverse components of both fields being considerably smaller.

The two classes of hydrodynamic and hydromagnetic disruption roughly correspond to the classes of models where $e_{\text {kin }}^{z}$ does or does not exceed $e_{\mathrm{mag}}$, respectively. If $e_{\mathrm{kin}}^{z}$ exceeds the volumeaveraged total magnetic energy density after reaching the saturation phase, deceleration enters the more efficient hydrodynamic regime. Otherwise, deceleration is caused by the magnetic field. A given model can undergo a transition from one class to the other one: for a weak initial field the early evolution may be dominated by 3D hydrodynamic turbulence, leading to an efficient deceleration of the shear flow and the magnetic field remaining at the same level; but when the kinetic energy of the turbulent flow decreases below that of the magnetic field, the deceleration rate drops to the hydromagnetic value.

Hence, we can summarize the influence of physical and numerical parameters on the turbulence and the deceleration as follows:

- Larger random perturbations favour 3D hydrodynamic instabilities. Comparing for $A=50$ a model with $\xi_{\text {rnd }}=10^{-2}$ and $\xi_{\text {rnd }}=10^{-4}$, we find significantly stronger magnetic fields and transverse velocities for the former model, indicating more vigorous turbulence and a faster deceleration of the shear flow.

- In 2D, weaker initial fields lead to slower deceleration, while 3D models exhibit a more complex dependence on the initial Alfvén number. As discussed above, hydrodynamic instabilities of the $\mathrm{KH}$ vortex tube dominate in case of very weak fields, leading to very rapid deceleration (Fig. 17). If the magnetic field is sufficiently strong, i.e., as long as $e_{\text {mag }}>e_{\text {kin }}^{z}$ holds, deceleration is initially similar to that in 2D for the same initial field strength, but drops strongly afterwards. Due to the deceleration, the $y$ and $z$-components of the velocity field reach equipartition.

- The dependence on the grid resolution is complementary to that on the initial Alfvén number. Finer grids allow for a more efficient field amplification, and thus favor hydromagnetic over hydrodynamic deceleration.

According to the 2D simulations, a maximum kinematic amplification is obtained for a sufficiently fine grid at a given initial field strength, i.e., increasing the grid resolution does not enhance the influence of the magnetic field. Thus, we expect an upper limit for the importance of magnetic vs. hydrodynamic deceleration corresponding to the upper limit of the field amplification. Even for infinite grid resolution, kinematic amplification of the magnetic field may not lead to a sufficiently fast field growth to compete with 3D hydrodynamic instabilities, if the initial field is too weak. Consequently, we anticipate only a weak dependence on the magnetic field for large initial Alfvén numbers.

Due to the lack of adequate numerical resolution in $3 \mathrm{D}$, we do not give any scaling laws for, e.g., $M_{x, y}^{\max }$ and $t_{\mathrm{dec}}$ as a function of the initial Alfvén number or the grid resolution.

\subsection{Supersonic shear flows}

Three-dimensional supersonic shear flows show pronounced differences with respect to 2D ones, the transverse kinetic energy densities growing much faster in 3D (see Fig. 19 for the evolution of a non-magnetized model). Furthermore, unlike for subsonic models, 3D hydrodynamic instabilities disrupt supersonic shear flows, i.e., they are not secondary instabilities feeding off a $\mathrm{KH}$ vortex tube. For the model shown in Fig. 19, we find that $e_{\text {kin }}^{z}$ grows at a rate similar to the $2 \mathrm{D}$ one only until $t \approx 20$ when it becomes comparable to $e_{\mathrm{kin}}^{y}$. Subsequently, both energy densities grow at the same rate, which is much faster than the corresponding $2 \mathrm{D}$ one.

The 3D instability prevents the shock-mediated formation of a $\mathrm{KH}$ vortex (Sect. 4.2). Instead of such a coherent large-scale flow, a rather turbulent flow forms at the shearing interface expanding in $y$-direction. Similarly to the 2D case, shocks develop at some distance from the interface, but these dissolve when engulfed by the turbulent flow. Unlike their 2D counterparts, they play no role in the development of the instability. During the 


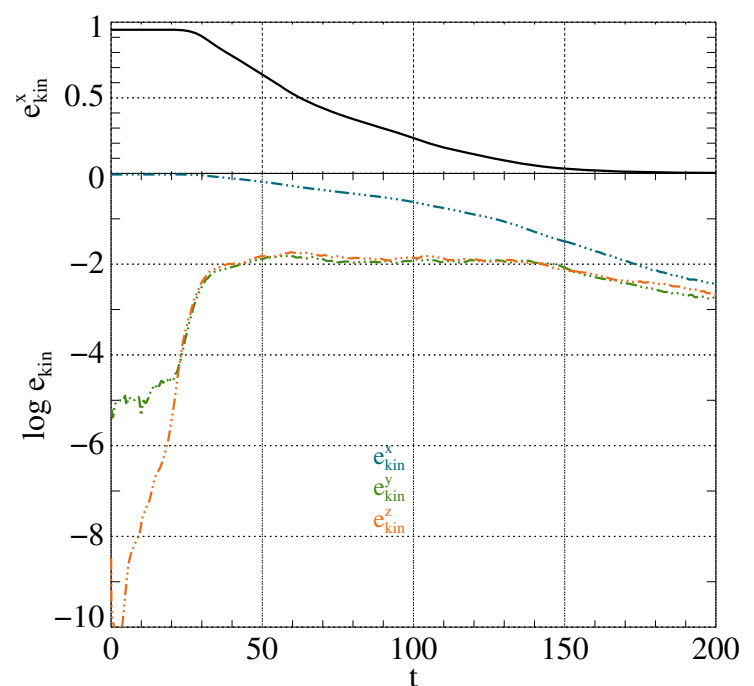

Fig. 19. Same as the top panel of Fig. 14, but for a model with $M=4$ and $b_{0}=0$.

saturation phase, the kinetic energy decreases due to efficient turbulent dissipation.

The interaction of shocks resulting from the usage of reflecting boundaries is essential for the growth of the instability in 2D. When open boundaries allow shocks to leave the computational domain, our 2D models are stable. In three dimensions, on the other hand, the instability does not depend on the presence of these shocks, i.e. the instability also grows when open boundaries are imposed (in $y$-direction). Hence, we find a good agreement between simulations of supersonic models computed with either type of boundary condition.

A weak initial magnetic field is amplified at the same rate as the kinetic energy when the instability develops. The exponential amplification ceases when the volume-averaged transverse kinetic energy densities $e_{\mathrm{kin}}^{y}$ and $e_{\mathrm{kin}}^{z}$ saturate (see Fig. 20). Afterwards ( $30 \lesssim t \lesssim 80$ ), we find only a very gradual growth of the magnetic energy. Typically, the volume-averaged transverse kinetic energy density, $e_{\mathrm{kin}}^{y z} \equiv e_{\mathrm{kin}}^{y}+e_{\mathrm{kin}}^{z}$, is reduced with respect to the non-magnetic case, but when adding the volume-averaged transverse magnetic energy density, $e_{\mathrm{mag}}^{y z}=e_{\mathrm{mag}}^{y}+e_{\mathrm{magn}}^{z}$, the total transverse energy density $e_{\mathrm{kin}}^{y z}+e_{\mathrm{mag}}^{y z}$ is at the same level as the transverse kinetic energy of a non-magnetized model.

The deceleration rate of the shear flow depends, as in the subsonic case, on the relative importance of hydrodynamic and hydromagnetic turbulence. There is, however, a physical difference to the subsonic case: the supersonic instability is dominated by strong 3D hydrodynamic turbulence already early on in the evolution, because it does not result from coherent 2D flows such as a KH vortex. Hence, there is no efficient kinematic amplification, and the magnetic field can become important only if it is maintained or slowly amplified by the 3D turbulence responsible, at the same time, for a decrease of the kinetic energy.

At an intermediate stage, $t=60$ (left panel of Fig. 21), the instability has not yet affected the entire computational volume in $y$-direction. Both the velocity and the magnetic field of that model exhibit a pronounced small-scale structure around the initial shearing layer. No preferred direction can be identified, and $e_{\mathrm{kin}}^{y z}>e_{\mathrm{mag}}^{y z}$. This has changed at $t=200$ (right panel), when due to efficient turbulent deceleration the total kinetic energy density has decreased by roughly an order of magnitude, similarly to the transverse magnetic energy $e_{\text {mag }}^{y z}$. The longitudinal

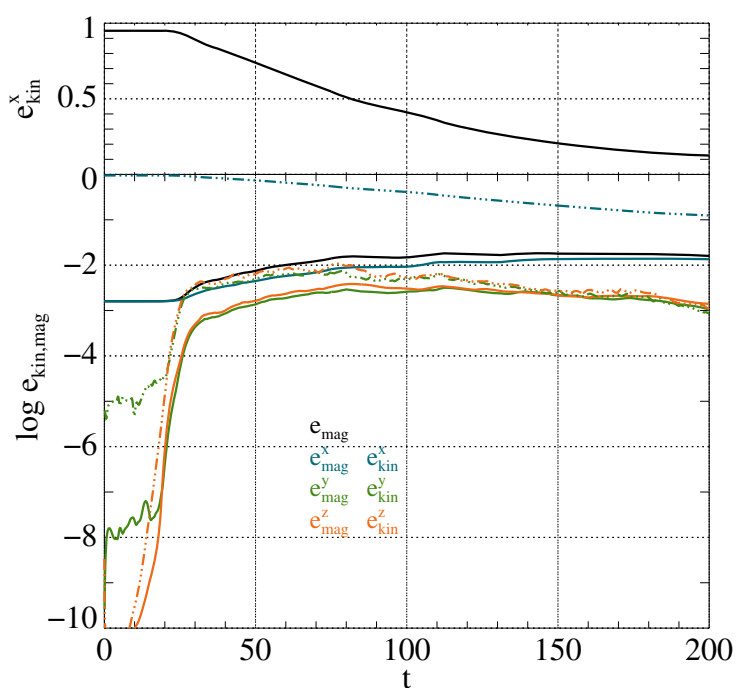

Fig. 20. Same as Fig. 15, but for a model with $M=4$ and $A=50$.

magnetic energy density $e_{\text {mag }}^{x}$, in contrast, has remained at the same level with $e_{\mathrm{mag}}^{x}>e_{\mathrm{mag}}^{y z}$. The dominance of $e_{\mathrm{mag}}^{x}$ and hence of $b^{x}$ exerts an ordering influence on the turbulent magnetic and velocity fields, enforcing an alignment of the flow with the field, similarly to the Alfvén effect of hydromagnetic turbulence. As a result, we find prominent coherent structures elongated in field direction.

\subsection{Anti-parallel magnetic field}

We have simulated a few of the models discussed above also using anti-parallel initial magnetic fields. With the total flux through surfaces $x=$ const. vanishing, the $x$-component of the magnetic field can decay to zero. This will particularly happen for weak fields. Stronger fields decay less efficiently because of resistive instabilities.

For a large initial random perturbation, the evolution is very similar to models with parallel initial fields. The shear flow is decelerated very efficiently, and kinetically dominated decaying turbulence with a very weak degree of anisotropy develops. Once the kinetic energy density approaches the magnetic one, the deceleration rate decreases. However, it does not tend to zero as in the parallel field case. Instead of leveling off at a constant value, both the kinetic and magnetic energy densities continue to decrease at a similar rate.

Models with a small initial random perturbation show, depending on the initial field strength, hydrodynamic or hydromagnetic deceleration. The field strength required for hydromagnetic to dominate over hydrodynamic deceleration is higher than for parallel fields. In several models we find at late stages the same evolution as described above: the kinetic and magnetic energy densities decay at a similar rate.

\section{Merger-motivated models}

After having discussed basic properties of magnetized shear layers, we now address simulations mimicking the conditions of shear layers arising in the merger of two magnetized neutron stars. We assume that the merging neutron stars heat up so much that any solid crust they may have developped during their 


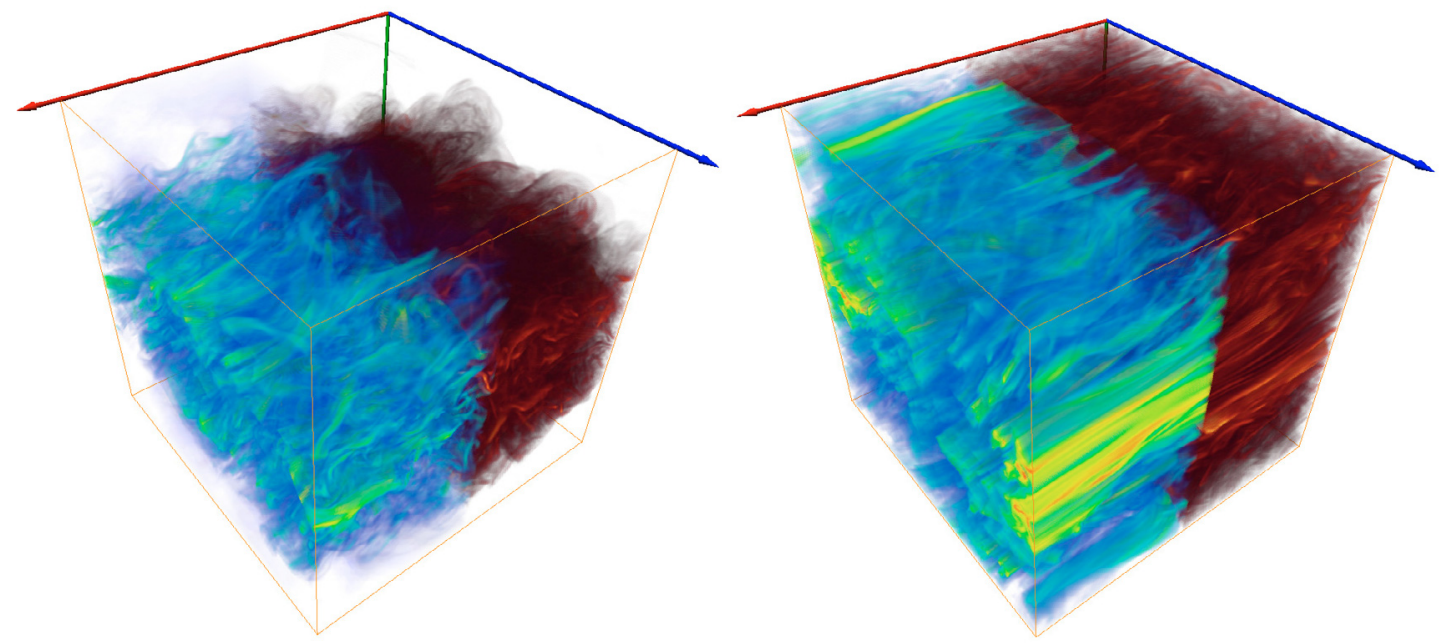

Fig. 21. Structure of a model with $\mathrm{M}=4$ and $\mathrm{A}=50$ (the same model as shown in Fig. 20) at $t=60$ (left panel) and $t=200$ (right panel). The two panels show the same variables as the ones in Fig. 18, i.e., the volume-rendered magnetic field strength (foreground, blue-green) and the modulus of the vorticity (background, red).

pre-merger evolution has melted, and the fluid approximation is valid in the shear layer.

\subsection{Physics, initial and boundary conditions}

\subsubsection{Equation of state}

We employed a simple parametrised equation of state to describe the thermodynamic properties of neutron star matter (Keil et al. 1996). This hybird equation of state assumes that the total gas pressure, $P$, is given by the sum of a barotropic part, $P_{\mathrm{b}}$, and a thermal part, $P_{\mathrm{th}}$ :

$P=P_{\mathrm{th}}+P_{\mathrm{b}} \equiv\left(\Gamma_{\mathrm{th}}-1\right) \varepsilon_{\mathrm{th}}+\kappa \rho^{\Gamma_{\mathrm{b}}}$,

where the thermal energy density, $\varepsilon_{\mathrm{th}}$, is given by the (total) energy density, $\varepsilon$, and the energy density of the polytropic component, $\varepsilon_{\mathrm{b}}$, according to

$\varepsilon^{\text {th }}=\varepsilon-\varepsilon_{\mathrm{b}}$.

The sound speed, required for the approximate Riemann solver and for the determination of the time step, is given by

$c_{\mathrm{s}}^{2}=\frac{\Gamma_{\mathrm{b}} P_{\mathrm{b}}+\Gamma_{\mathrm{th}} P_{\mathrm{th}}}{\rho}$.

We used $\Gamma_{\mathrm{b}}=\Gamma_{\text {th }}=1.333$, appropriate for dense matter whose pressure is dominated by relativistically degenerate electrons.

\subsubsection{Initial conditions}

With presently available computational resources it is not possible to perform global simulations of the close encounter or merging of two magnetized neutron stars with a grid resolution sufficiently high to resolve also the growth of $\mathrm{KH}$ instabilities in shearing magnetized neutron star matter. Nevertheless one can study some aspects of this phenomenon by means of local simulations covering only a small volume around the shear layer.

To this end we consider a quadratic (2D) / cubic (3D) computational domain in Cartesian coordinates assuming that the $x$-axis is parallel to the direction of the shear flow, the $y$-axis parallel to the line connecting the centers of the two neutron stars, and the $z$-axis (in 3D) perpendicular to that line. As the edges of our computational domain have a size of $200 \mathrm{~m}$ only, i.e., they are much smaller than the radius of a neutron star, we consider only homogeneous initial states, i.e, initial models with constant density and pressure. Besides the shear flow in $x$-direction the initial models are static, too. This approximation is justified as the merging neutron stars move much faster in $x$-direction than they approach each other in $y$-direction due to the action of gravity. Accordingly, we use periodic boundary conditions in $x$ and $z$-direction, and reflecting ones in $y$-direction.

The shear velocity $v_{x}$, corresponding to either a Mach number of $M=1$ or $M=4$, has the same tanh-profile as that used in the simulations of the previous sections, and we also consider both parallel and anti-parallel initial magnetic field configurations. The shear velocity is supposed to mimic the orbital velocity of the two neutron stars. We trigger the instability by applying similar perturbations as in the previous sections, i.e., a combination of a sinusoidal and a random velocity perturbation.

\subsection{Two-dimensional models}

A number of models (see Table A.6) computed in two dimensions confirm the basic results discussed in the previous sections, i.e., the occurrence of three phases, namely $\mathrm{KH}$ growth, kinematic amplification, and saturation. This also holds for the dependence of the parameters characterizing these phases, e.g., the termination values of the field strength and magnetic energ, on the initial data and the grid resolution.

We performed simulations with up to $2048^{2}$ zones. The width of the shear layer was $a=10 \mathrm{~m}$, and the initial velocity $v_{0}^{x}=1.83$ or $7.2 \times 10^{9} \mathrm{cms} / \mathrm{s}$, for models with $\mathrm{M}=1$ or $\mathrm{M}=4$, respectively. Due to the affordable grid resolution we employed rather strong initial fields of the order of $b_{0}^{x} \sim 10^{14} \mathrm{G}$, corresponding to Alfvén numbers $\mathrm{A} \approx 115\left(10^{14} \mathrm{G} / b_{0}^{x}\right)$. The initial field was either parallel or anti-parallel to the shear flow.

We start the discussion with models with $\mathrm{M}=1$. The KH instability developed within less than $0.05 \mathrm{~ms}$, establishing one large $\mathrm{KH}$ vortex. Afterwards, the magnetic field is amplified kinematically by the vortical flow. The physics of KH growth termination is the same as that described in Sect. 4.3. Hence, we 
also find a similar dependence for the field amplification factor $f_{\text {kin }}$ on the initial field strength and the grid resolution.

- On finer grids one can resolve the increasingly thin structures of the magnetic field better. Consequently, one finds more efficient amplification, until for a sufficiently fine grid convergence of the amplification factor is achieved.

- Weaker initial fields are amplified by a larger amount, i.e., the maximum value of the field strength at the end of the KH growth phase depends only weakly on the initial field (assuming numerical convergence). The total magnetic energy increases with increasing initial field strength due to the larger volume filling factor of magnetic flux tubes for stronger initial fields.

After termination of the kinematic amplification phase, the topology of the subsequent turbulent saturation phase is dominated by a multitude of thin flux sheets. Due to deceleration by magnetic stresses, the kinetic energy of the shear flow decreases at a rate depending on the initial field strength. Lacking a driving force, the turbulence decays gradually. At late stages it is dominated by the parallel component of the magnetic field $b^{x}$, leading to a strong alignment of the flux sheets in $x$-direction.

Models with $b_{0}^{x}=5, \times 10^{13} \mathrm{G}$ and $10 \times 10^{13} \mathrm{G}$ reach slightly fluctuating maximum field strengths around $3 \times 10^{15} \mathrm{G}$ in the saturated state. The volume filling factor of the magnetic field, i.e., the relative volume occupied by intense magnetic flux tubes, decreases with decreasing initial field strength leading to a weaker mean magnetic field and consequently a slower deceleration of the shear flow for weaker initial fields. We find mean fields of $\sim 5 \times 10^{14} \mathrm{G}$ and $\sim 2.5 \times 10^{14} \mathrm{G}$ for $b_{0}^{x}=10^{14} \mathrm{G}$ and $b_{0}^{x}=5 \times 10^{13} \mathrm{G}$, respectively. The time scale for deceleration of the shear flow is less than 1 millisecond. For a model with an initial field of $2 \times 10^{14} \mathrm{G}$, the deceleration is sufficiently rapid to cause a significant decay (by about an order of magnitude) of the turbulent energy within $0.5 \mathrm{~ms}$.

The evolution of the shear layer is affected by the choice of the initial field configuration. Parallel initial fields have, similarly to our observations above, a somewhat larger impact on the dynamics of the $\mathrm{KH}$ instability. In this case, the non-vanishing magnetic flux threading surfaces $x=$ const. is conserved due to the boundary conditions, and gives rise to an effective driving force. Apart from lacking this additional driver, anti-parallel magnetic fields are prone to stronger dissipation due to presence of stronger currents at the boundaries between regions of opposite magnetic polarity.

The evolution of models with a supersonic shear flow $(\mathrm{M}=$ 4 ) is similar to that of their dimensionless conterparts discussed previously. With initial fields between 10 and $40 \times 10^{13} \mathrm{G}$, the initial Alfvén numbers of the shear flow are between $\sim 110$ and $\sim 440$, i.e., in the range covered in Sect. 4.4. The dynamics is the same: pressure waves steepen into oblique shocks, and the dissipation of kinetic into thermal energy in these shocks creates a broad transition layer between the two regions of positive and negative $v_{x}$. The shear flow is decelerated very efficiently even for very weak fields. We find 3field amplification up to $5 \ldots 10 \times$ $10^{15} \mathrm{G}$ for the maximum field strength and $1 \ldots 2 \times 10^{15} \mathrm{G}$ for the volume-averaged rms value of the field, $\sqrt{1 / \mathcal{V} \int \mathrm{d} \mathcal{V} \boldsymbol{b}^{2}}$, i.e., of the same order as in the case $M=1$ but systematically higher by a factor $\sim 2$, with considerably higher values for parallel than for anti-parallel initial fields.

Hence, the results and in particular their dependence on the physical and numerical parameters of the models explored in Sect. 4 , are robust with respect to the described variations of the initial conditions. Consequently, we can expect them to apply to merger systems without too strong modifications.

\subsection{Three-dimensional models}

One of the main questions to be addressed by $3 \mathrm{D}$ simulations is whether the dynamics of these models is dominated by magnetic flux tubes or by 3D hydrodynamic instabilities. As we have seen in the previous sections, this has a distinct influence on, e.g., the magnetic field strength achieved at saturation.

For the 3D simulations we used grids of up to $m_{x} \times m_{y} \times m_{z}=$ $256^{3}$ zones. The initial field strength was between 5 and $40 \times$ $10^{13} \mathrm{G}$. We again applied different combinations of sinusoidal and random velocity perturbations to the shear layer.

The models (see Table A.7) show the same overall dynamics and the same evolutionary phases as the corresponding models discussed in Sect. 4. We find the initial KH growth phase, the kinematic amplification phases, followed by the development of parasitic instabilities leading to a non-linear saturated state. The flow during the first two phases is very similar to that in $2 \mathrm{D}$, and field amplification follows the same trends with initial field and grid resolution as outlined above. The further evolution depends, as discussed above, strongly on the relative amplitude of random and sinusoidal perturbations.

When a small random perturbation is imposed, field amplification proceeds through the first two growth phases the field strength being limited by its back reaction onto the flow. These models suffer (if resolved well on a sufficiently fine grid) hydromagnetic instabilities of the flux sheet, leading to the break-up of the KH vortex tube and the deceleration of the shear flow. For a well-resolved model with $b_{0}^{x}=2 \times 10^{13} \mathrm{G}$ the maximum magnetic field strength is $\approx 9 \times 10^{15} \mathrm{G}$, while the rms maximum is only $\approx 9 \times 10^{14} \mathrm{G}$.

For models with large random perturbations and for models with very weak initial fields the disruption of the $\mathrm{KH}$ vortex tube is predominantly due to hydrodynamic instabilities leading to a very efficient deceleration of the shear flow. These instabilities grow on a very short time scale, causing a strong growth of the volume-averaged transverse kinetic energy densities $e_{\text {kin }}^{y}$ and $e_{\mathrm{kin}}^{z}$, as well as of all volume-averaged magnetic energy densities $\left(e_{\mathrm{mag}}^{x}, e_{\mathrm{mag}}^{y}\right.$, and $\left.e_{\mathrm{mag}}^{z}\right)$.

The amplification factors are similar for all components of the field, leading to equipartition among them at peak magnetic energy. The amplification rate is at first very large but decreases strongly as the parasitic instabilities saturate. Eventually, the magnetic energy reaches a maximum, and then starts to decrease again. This maximum depends either on the grid resolution (for the most weakly magnetized models), or on the dynamic backreaction of the field onto the flow. In the latter case, field amplification ceases once the volume-averaged transverse kinetic energy densities (decaying from their maximum values at saturation of the parasitic instabilities) decrease to roughly the level of the volume-averaged magnetic energy density.

The magnetic field is amplified during all three growth phases: at the $\mathrm{KH}$ growth rate during the $\mathrm{KH}$ growth phase, at a (smaller) rate determined by the overturning velocity of the $\mathrm{KH}$ vortex tube during the kinematic amplification phase, and during the growth of the parasitic instabilities. Since the magnetic field starts to decrease shortly after saturation of the parasitic instabilities feeding off the shear flow, the maximum field strength is reached at that moment. The magnetic field energy can reach at most equipartition with the (decaying) transverse kinetic energy, which typically has a value of $\sim 10^{43} \mathrm{erg}$. For a 

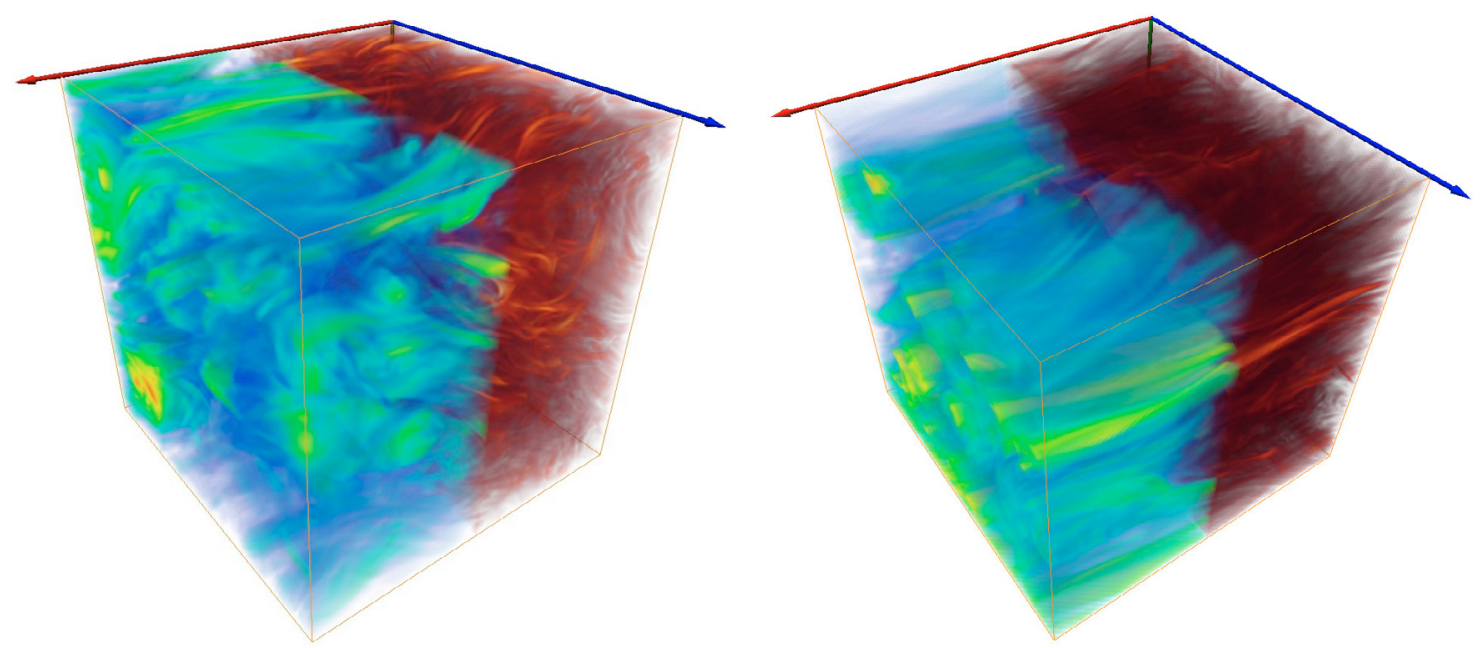

Fig. 22. 3D structure of the final turbulent state of models with $b_{0}^{x}=5 \times 10^{13} \mathrm{G}$ (left panel) and $b_{0}^{x}=20 \times 10^{13} \mathrm{G}($ right panel) at $t=1 \mathrm{~ms}$, respectively. The panels show the volume-rendered magnetic field strength (front half; blue-green-yellow-red corresponding to increasing values of $|\boldsymbol{b}|$ ) and enstrophy (rear half; red-yellow corresponding to increasing values of $\left.(\boldsymbol{\nabla} \times \boldsymbol{v})^{2}\right)$. The red and blue long arrows mark the $x$ and $z$-direction, respectively.

model with $b_{0}^{x}=4 \times 10^{14} \mathrm{G}$ the corresponding root mean square saturation field is $\sim 1.6 \times 10^{15} \mathrm{G}$. The weaker the initial field, the smaller is the maximum magnetic energy, since the achievable amplification factor is limited by the duration of the three field amplification phases. The maximum field strength reached anywhere in the computational domain depends only weakly on the initial field, and has a value between 6 and $10 \times 10^{15} \mathrm{G}$.

The decay of the turbulence (measured by the transverse kinetic and magnetic energy densities) as well as that of the shear flow starts at a similar rate for all models, and the magnetic field decreases much faster than it does in the corresponding 2D models. Shortly after $(\sim 0.05 \mathrm{~ms})$ the rms field strength as well as the total field strength reach their peak values early during the saturation phase, the kinetic energy densities decay very rapidly, and much faster than the magnetic energy density. The decay slows down shortly after $e_{\text {kin }}^{y}+e_{\text {kin }}^{z}$ has decreased below the value of $e_{\text {mag. }}$. Afterwards, all transverse energy densities decay at a similar rate. In the more strongly magnetized models this happens when $e_{\text {kin }}^{x}$ (which can undergo a phase of particularly fast decay) is still larger than $e_{\mathrm{kin}}^{y}+e_{\mathrm{kin}}^{z}$, whereas it is usually the other way round for weaker initial fields (a similar effect can be observed for under-resolved models where insufficient grid resolution limits the field amplification). During the further evolution the relative sizes of $e_{\mathrm{kin}}^{x}, e_{\mathrm{kin}}^{y}$, and $e_{\mathrm{kin}}^{z}$ remain unchanged.

For weak initial fields, $\left(b_{0}^{x}=1,5\right.$, and $10 \times 10^{13} \mathrm{G}$, the kinetic energy density dominates the magnetic one by a factor $\approx 2.7$ in the final state. Concerning the volume-averaged kinetic energy densities we find $e_{\mathrm{kin}}^{x} \approx e_{\mathrm{kin}}^{z} \approx 2.4 e_{\mathrm{kin}}^{y}$. This relation also holds for the volume-averaged magnetic energy densities, indicating a relatively high degree of isotropy of the turbulence. The final state for $b_{0}^{x}=5 \times 10^{13} \mathrm{G}$ is shown in the left panel of Fig. 22 . Obviously, neither the flow nor the magnetic field show any preferred direction. Instead, one recognizes a complex pattern of tangled small-scale flux tubes.

For sufficiently strong initial fields $\left(b_{0}^{x}=20\right.$, and $40 \times$ $\left.10^{13} \mathrm{G}\right)$, the final state is more strongly magnetized. As the turbulent energy decays more rapidly than the $x$-component of the magnetic field, $b_{x}$ dominates the dynamics after $t \approx 15 \mathrm{~ms}$ leading to a slower deceleration of the shear flow and a more pronounced alignment of flow features (flux and vorticity tubes) in $x$-direction (see Fig. 22, right panel).

Similarly to the 2D models discussed above, a parallel initial magnetic field has a stronger influence on the dynamics than an anti-parallel one: the field strength reaches a higher maximum value, and the influence of hydrodynamic instabilities is slightly less. At late stages, such models may exhibit a phase of hydromagnetic deceleration, in contrast to the roughly constant value of $e_{\text {kin }}^{x}$ in models with strong anti-parallel initial fields.

For of supersonic shear flows with $\mathrm{M}=4$, the evolution is similar to that of the dimensionless models discussed in Sect. 5.2. We find a fast growth of 3D hydrodynamic instabilities disrupting the shear flow before the shock-mediated mechanism working in 2D can operate. Turbulence sets in quickly without the intermediate development of a KH vortex, and the shear flow is decelerated very efficiently. The maximum magnetic fields we find are of the order of $1 \times 10^{16} \mathrm{G}$ for the absolute maximum, and $3 \times 10^{15} \mathrm{G}$ for the rms field, respectively. These values are rather insensitive to the initial field strength and geometry.

\section{Summary and conclusions}

Global simulations indicate that the contact layer between two merging neutron stars is a site of very efficient field amplification. The layer is prone to the Kelvin-Helmholtz instability, and thus, exponential growth of any weak seed field is possible, as observed by Price \& Rosswog (2006) (see also Giacomazzo et al. 2009; Anderson et al. 2008; Liu et al. 2008). The limitations of their simulations, mainly concerning grid resolution, did not allow these authors to determine the saturation level of the instability firmly and accurately. Thus, the implications of magnetic fields for the merger dynamics remains unclear. On the basis of energetic arguments, the instability might lead to a field in equipartition with the kinetic or the internal energy of the shear flow, corresponding to field strengths of the order of $10^{16} \mathrm{G}$ or $10^{18} \mathrm{G}$, respectively.

We reassessed these arguments by means of local highresolution simulations of magnetized shear layers in two and 
three spatial dimensions. To this end we performed more than 220 simulations focusing on properties of the hydromagnetic $\mathrm{KH}$ instability in general as well as on the contact surfaces of merging neutron stars. We refer to these two classes of simulations as dimensionless and merger-motivated models, respectively.

We employed a recently developed multi-dimensional Eulerian finite-volume ideal MHD code based on high-order spatial reconstruction techniques and Riemann solvers of the MUSTA-type (Obergaulinger 2008; Obergaulinger et al. 2009).

We set up a KH-unstable shear flow in Cartesian coordinates in a quadratic (2D) and cubic (3D) computational domain imposing periodic boundary conditions in the direction of the shear flow and reflecting or open ones in the transverse directions. Focusing on the effects of a magnetic field on the instability, we used a simplified equation of state (ideal gas EOS and a hybrid barotropic/ideal gas EOS for the dimensionless and the merger-motivated models, respectively) and neglected additional physics, e.g., such as neutrino transport.

Under these simplifications, the shear flows are characterized by two parameters, the initial Mach number $M$ and the initial Alfvén number $A$, measuring the magnitude of the jump in shear velocity in units of the sound speed and the Alfvén velocity, respectively.

Analytic considerations and previous simulations of nonmagnetized shear flows show that the growth rate of the $\mathrm{KH}$ instability as well as its saturation level (i.e., the kinetic energy of the circular $\mathrm{KH}$ vortex formed by the instability) increase with increasing $\mathrm{M}$ for subsonic shear flows . A magnetic field is known to reduce the growth rate and potentially, i.e., for $A<2$, even to suppress the instability (Chandrasekhar 1961; Miura \& Pritchett 1982; Keppens et al. 1999). However, less is known about the saturation level and the dynamic back-reaction, in particular for weak initial fields.

Frank et al. (1996); Jones et al. (1997); Jeong et al. (2000); Ryu et al. (2000) studied the evolution of the hydromagnetic KH instability in two and three dimensions. In 2D, models undergo a transition from non-linear stabilization of the $\mathrm{KH}$ vortex to its violent disruption or more gradual dissipation when the initial field strength is reduced, while in 3D purely hydrodynamic elliptic instabilities of the vortex tube may dominate over MHD effects.

The study of these non-linear effects is hampered by high requirements on the grid resolution that is necessary to follow the development of increasingly thin magnetic flux sheets and tubes. This limits the range of Alfvén numbers for which numerical convergence can be achieved to rather modest values. It also reduces the predictive power for merger systems, where rather weak initial fields are expected. This limitation can be overcome only when using large grids in combination with a highly accurate code. We evolved subsonic, transsonic, and supersonic shear flows with $M \in[0.5 ; 1 ; 4]$, while using the maximum Alfvén numbers for which convergence is achievable. The resulting broad range of Alfvén numbers covered by our simulations allows us to establish scaling laws governing the field amplification as a function of the initial field strength.

The main results of our simulations are:

1. In $2 \mathrm{D}$, we confirm the results of analytic work (in the linear regime) and previous simulations concerning the growth rate and the saturation of the transverse kinetic energy densities for strong initial fields due to Chandrasekhar (1961); Miura \& Pritchett (1982); Keppens et al. (1999). This agreement supports the viability of our numerical approach for the problem at hand.
2. For subsonic shear flows $(\mathrm{M}=0.5,1)$ we explored a wide range of initial field strengths covering Alfvén numbers up to $\mathrm{A}=5000$ in $2 \mathrm{D}$.

(a) For intermediate and weak fields, we distinguish two phases: the $\mathrm{KH}$ growth phase during which the field grows at the $\mathrm{KH}$ growth rate, and after formation of a $\mathrm{KH}$ vortex, a phase of kinematic field amplification by the overturning vortex. The growth rate during the latter phase depends on the velocity of the vortex. The field is highly intermittent and concentrated in flux sheets, which are stretched by the flow leading to an exponential growth of the field strength while the sheet width decreases.

(b) The termination of the kinematic amplification phase occurs either numerically, when the flux sheets get too thin to be resolved on a given computational grid, or dynamically by back-reaction of the field onto the flow. The most important mode of back-reaction is the growth of secondary resistive instabilities feeding off the magnetic energy of the flux sheets. These instabilities terminate the kinematic field growth and initiate the non-linear saturation phase during which the $\mathrm{KH}$ vortex is destroyed by the ensuing MHD turbulence and the shear flow is gradually decelerated. This scenario is equivalent to that of the disruption models of Frank et al. (1996).

(c) We quantified the amount of field amplification during the kinematic amplification phase by computing the ratio of the volume-averaged Maxwell stress component $M_{x y}$ at the beginning and at the end of that phase. The amplification factor scales with the initial Alfvén number as $A^{3 / 4}$, corresponding to a scaling of the maximum Maxwell stress with the initial field strength as $b_{0}^{5 / 4}$. If the simulation is under-resolved, the amplification factor is reduced by a factor $\propto m^{7 / 8}$ ( $m$ being the number of zones per dimension). The maximum local field strength corresponds to a local equipartition between the magnetic energy density of a flux sheet and the kinetic energy density of the shear flow; it depends only weakly on the initial field.

(d) The secondary resistive instabilities observed in our simulations are triggered by numerical resistivity instead of a physical one. The numerical resistivity, which is a function of the grid resolution $\Delta$, is important only for small thin structures having a spatial size of the order of $\Delta$ or less. In our simulations, it causes current sheets to become unstable when their width approaches the grid spacing $\Delta$. Although only simulations with arbitrarily high resolution can sustain arbitrarily thin and intense current sheets, we observe nevertheless convergence: the field amplification becomes independent of the grid resolution, if $\Delta$ is smaller than some threshold which depends on the initial field strength. The reason for this independence is the fact that the most unstable current sheets do not consist of individual flux sheets but of pairs or triples of coalescing flux sheets. Thus, decreasing the distance between flux sheets does not lead to a stronger field (which would be the case, if a single flux sheet is compressed in transverse direction).

(e) The disruption of the vortex and the efficient dissipation set these resisto-dynamic models apart from the class of dissipation models with even weaker initial fields where the KH vortex remains intact, and only very slow dissipation is provided by turbulence. In the simulations of Frank et al. (1996), secondary instabilities do not modify 
the flow field qualitatively. Our simulations indicate that this is, partially at least, a resolution effect. If a simulation is under-resolved and the field growth is not limited by dynamic back-reaction but by the resolvable width of flux sheets, no disruption will occur, and the deceleration time of the shear flow is very long. Converged simulations show, on the other hand, the disruption of the $\mathrm{KH}$ vortex by secondary magnetic instabilities when the magnetic field strength approaches a local maximum close to equipartition with the kinetic energy density of the shear flow. This happens in all converged models, but for weak initial fields, the deceleration time can be very long.

(f) Models with initially anti-parallel and parallel magnetic fields, but otherwise identical, give qualitatively similar results, the above discussed effects being somewhat less pronounced in case of the former field configuration.

3. The contact layer of merging neutron stars resembles supersonic shear flows. In principle, these are stable. We find, however, that an exponentially growing instability may occur when closed boundary conditions are imposed in the direction transverse to the shear flow. The instability is mediated by shock waves traveling through the computational domain. The corresponding growth rates are much smaller than for subsonic shear flows. The effects of a magnetic field on a supersonic shear flow are qualitatively similar to those on subsonic shear flows.

4. In 3D the disruption of the $\mathrm{KH}$ vortex tube can be induced by a purely hydrodynamic secondary so-called elliptic instability as discussed, e.g., by Ryu et al. (2000). It leads to a very rapid growth of the kinetic energy densities corresponding to all components of the flow velocity once the $\mathrm{KH}$ vortex tube forms, and decelerates the shear flow more efficiently than the MHD mechanisms outlined above. Which of the two possible disruption mechanisms, elliptic or hydromagnetic, operates depends on the initial field strength $b_{0}$ and the value of volume-averaged kinetic energy density $e_{\text {kin }}^{z}$. The magnetic mechanism will dominate only if $e_{\mathrm{mag}}>e_{\mathrm{kin}}^{z}$, i.e., as long as the magnetic energy density exceeds the transverse kinetic energy in z-direction. Due to the very fast growth of the elliptic instability, this may be the case only for a short time, if at all. A rather strong initial field and small perturbations in $z$-direction are required for a hydromagnetic disruption.

5. $2 \mathrm{D}$ and $3 \mathrm{D}$ simulations of shear flows with merger-motivated initial conditions performed in a cubic computational domain of constant density and pressure having an edge size of $200 \mathrm{~m}$ show the same overall dynamics as corresponding dimensionless models. The initial Mach number of the shear flow was chosen to be $M=1$ and $M=4$ corresponding to a density of $10^{13} \mathrm{~g} \mathrm{~cm}^{-3}$, and shear velocities of $1.83 \times 10^{9} \mathrm{~cm} / \mathrm{s}$, and $7.2 \times 10^{9} \mathrm{~cm} / \mathrm{s}$, respectively. The initial magnetic field strength was varied between $5 \times 10^{13} \mathrm{G}$ and $4 \times 10^{14} \mathrm{G}$.

(a) The instability grows rapidly: saturation occurs within $\lesssim 0.1 \mathrm{~ms}$, and the disruption and deceleration times are much less than $1 \mathrm{~ms}$.

(b) The dynamics is the same as that of the dimensionless models. Field amplification leads to a maximum field strength $\lesssim 10^{16} \mathrm{G}$, and a rms value of $\lesssim 1.6 \times 10^{15} \mathrm{G}$. These values are the same for $3 \mathrm{D}$ models suffering hydrodynamic and hydromagnetic disruption.

From our results, we may draw a few conclusions concerning the growth and the influence of magnetic fields in neutron-star mergers. The foremost implication is that the maximum field strength, independent whether it refers to a single point or a spatial average, is not amplified to equipartition with the thermal energy density. We can, hence, exclude saturation fields of the order of $10^{18} \mathrm{G}$ in the contact layers of neutron star mergers.

Instead, local equipartition with the kinetic energy density is reached with corresponding maximum fields $\sim 10^{16} \mathrm{G}$, as speculated by Price \& Rosswog (2006). Due to the high degree of intermittency in the case of weak initial fields, the (rms) average of the field strength is smaller, i.e, its direct dynamic impact (e.g., disruption of the $\mathrm{KH}$ vortex tube or deceleration of the shear flow) on the flow is probably rather limited. This is even more the case if the geometry of the system and the perturbations resulting from the merger dynamics enhance the importance of purely hydrodynamic instabilities. More indirect effects can, however, not be excluded, e.g., whether magnetic flux tubes created at the shear layer are transported rapidly far away by large-scale flows. The short period of time during which the magnetic field stays close to its maximum value and its fast decay impose severe constraints on the impact that the amplified fields may have on any hydromagnetic or electromagnetic jet-launching mechanism in a merger of two neutron stars. We note that magnetically driven relativistic outflows may need much longer time scales ( $\sim \mathrm{a}$ few $\mathrm{ms}$ ) to tap the rotational energy of either the black hole or the accretion disk resulting after the merger.

Though these results limit the prospect for magnetic effects to play a dynamic role in neutron star mergers, their proper inclusion in current and forthcoming simulations may be necessary, because magnetic fields influence the dissipation rates in the shear layer, i.e., their neglect may lead to an underestimation of the temperature in the shear layer, and hence in the accretion disk. Given the resolution requirements imposed by weak initial fields, a more sophisticated treatment of the problem probably also has to abandon the assumption of ideal MHD and to consider the formulation of a turbulence model for unresolved magnetic field structures.

Acknowledgements. This research has been supported by the Spanish Ministerio de Educación y Ciencia (grants AYA2007-67626-C03-01, CSD2007-00050), and by the Collaborative Research Center on Gravitational Wave Astronomy of the Deutsche Forschungsgemeinschaft (DFG SFB/Transregio 7). MAA is a Ramón y Cajal fellow of the Ministerio de Educación y Ciencia. Most of the simulations were performed at the Rechenzentrum Garching (RZG) of the MaxPlanck-Society. We are also thankful for the computer resources, the technical expertise, and the assistance provided by the Barcelona Supercomputing Center - Centro Nacional de Supercomputación. Parts of this article have been written during M.O.'s visit to the Departamento de Astronomía y Astrofísica of the Universidad de Valencia. He wants to express his gratitude for the kind hospitality experienced there.

\section{Appendix A: Tables of models}

We provide tables listing the parameters and important properties of the models computed:

Table A.1 lists the parameters of models which we computed to compare the growth rates obtained numerically with theoretical predictions, serving as code validation.

Table A.2 lists 2D hydrodynamic models of transonic and supersonic shear flows.

Tables A.3 and A.4 list the amplification factors of the magnetic field and the disruption and deceleration rates of models with weak initial fields, respectively.

Table A.5 lists the initial data of 3D dimensionless models.

Tables A.6 and A.7 list the initial conditions of 2D and 3D merger-motivated models, respectively. 
A\&A 515, A30 (2010)

Table A.1. Summary of models computed to compare numerical growth rates with theoretical predictions.

\begin{tabular}{lccccccccccc}
\hline \hline Name & $l_{x}$ & $l_{y}$ & $m_{x} \times m_{y}$ & $P_{0}$ & $U_{0}$ & $\mathrm{M}$ & $a$ & $\boldsymbol{b}_{0}$ & $k_{x}$ & $\Gamma_{\mathrm{MP}}$ & $\Gamma_{\text {num }}$ \\
\hline grw-1 & 1 & 2 & $50 \times 100$ & 1 & 1.29 & 1 & 0.05 & $(0,0,0)$ & $2 \pi$ & 1.73 & 1.64 \\
grw-2 & 1 & 2 & $100 \times 200$ & 1 & 1.29 & 1 & 0.05 & $(0,0,0)$ & $2 \pi$ & 1.73 & 1.74 \\
grw-3 & 1 & 2 & $200 \times 400$ & 1 & 1.29 & 1 & 0.05 & $(0,0,0)$ & $2 \pi$ & 1.73 & 1.75 \\
grw-4 & 1 & 2 & $400 \times 800$ & 1 & 1.29 & 1 & 0.05 & $(0,0,0)$ & $2 \pi$ & 1.73 & 1.75 \\
grw-5 & 1 & 2 & $200 \times 400$ & 1 & 1.29 & 1 & 0.025 & $(0,0,0)$ & $2 \pi$ & 2.4 & 2.44 \\
grw-6 & 1 & 2 & $200 \times 400$ & 1 & 1.29 & 1 & 0.1 & $(0,0,0)$ & $2 \pi$ & 0.66 & 0.68 \\
grw-7 & 1 & 2 & $200 \times 400$ & 1 & 0.645 & 0.5 & 0.05 & $(0,0,0)$ & $2 \pi$ & 1.09 & 1.07 \\
grw-8 & 1 & 2 & $200 \times 400$ & 1 & 1.843 & $10 / 7$ & 0.05 & $(0,0,0)$ & $2 \pi$ & 1.77 & 1.79 \\
grw-9 & 1 & 2 & $200 \times 400$ & 1 & 0.645 & 0.5 & 0.05 & $(0,0,0)$ & $4 \pi$ & 1.36 & 1.35 \\
grw-10 & 1 & 2 & $200 \times 400$ & 1 & 1.29 & 1 & 0.05 & $(0.129,0,0)$ & $2 \pi$ & 1.69 & 1.70 \\
grw-11 & 1 & 2 & $200 \times 400$ & 1 & 1.29 & 1 & 0.05 & $(0.258,0,0)$ & $2 \pi$ & 1.56 & 1.54 \\
\hline
\end{tabular}

Notes. The columns give the model name, the size of the domain $\left(l_{x}, l_{y}\right)$, the initial pressure, $P_{0}$, the velocity shear, $U_{0}$, the corresponding Mach number $\mathrm{M}=U_{0} / c_{\mathrm{s}}$, the initial magnetic field $\boldsymbol{b}_{0}$, the initial width of the shear flow, $a$, the corresponding wave number, $k_{x}$, the growth rate, $\Gamma_{\mathrm{MP}}$, obtained from Miura \& Pritchett (1982), and an estimate of the numerical growth rate, $\Gamma_{\text {num. }}$.

Table A.2. Summary of 2D hydrodynamic supersonic models.

\begin{tabular}{lccccccccccc}
\hline \hline Name & $l_{x}$ & $l_{y}$ & $m_{x} \times m_{y}$ & $P_{0}$ & $U_{0}$ & $\mathrm{M}$ & $a$ & $k_{x}$ & $\mathrm{BC}$ & $\Gamma_{\text {num }}$ & Oscillations \\
\hline HD2o-1-1 & 1 & 4 & $200 \times 800$ & 1 & 2.322 & 1.8 & 0.05 & $2 \pi$ & open & 0.97 & \\
HD2o-1 & 1 & 2 & $200 \times 400$ & 1 & 2.322 & 1.8 & 0.05 & $2 \pi$ & open & 0.96 & \\
HD2o-1-i & 1 & 1 & $200 \times 200$ & 1 & 2.322 & 1.8 & 0.05 & $2 \pi$ & open & 0.73 & \\
HD2o-1-s & 1 & 0.5 & $200 \times 100$ & 1 & 2.322 & 1.8 & 0.05 & $2 \pi$ & open & 0.16 & $\sqrt{ }$ \\
HD2o-2 & 1 & 2 & $200 \times 400$ & 1 & 2.451 & 1.9 & 0.05 & $2 \pi$ & open & 0.30 & $\sqrt{ }$ \\
HD2o-3 & 1 & 2 & $200 \times 400$ & 1 & 2.5155 & 1.95 & 0.05 & $2 \pi$ & open & 0.26 & $\sqrt{ }$ \\
HD2o-4 & 1 & 2 & $200 \times 400$ & 1 & 2.58 & 2 & 0.05 & $2 \pi$ & open & 0 & \\
HD2o-5 & 1 & 2 & $200 \times 400$ & 1 & 5.16 & 4 & 0.05 & $2 \pi$ & open & 0 & \\
HD2r-0 & 1 & 2 & $200 \times 400$ & 1 & 1.29 & 1 & 0.05 & $2 \pi$ & reflecting & 1.73 & \\
HD2r-1 & 1 & 2 & $200 \times 400$ & 1 & 2.322 & 1.8 & 0.05 & $2 \pi$ & reflecting & 0.96 & \\
HD2r-1-i & 1 & 1 & $200 \times 200$ & 1 & 2.322 & 1.8 & 0.05 & $2 \pi$ & reflecting & 0.56 & \\
HD2r-1-s & 1 & 0.5 & $200 \times 100$ & 1 & 2.322 & 1.8 & 0.05 & $2 \pi$ & reflecting & 0.56 & $\sqrt{ }$ \\
HD2r-1-S & 1 & 0.25 & $200 \times 50$ & 1 & 2.322 & 1.8 & 0.05 & $2 \pi$ & reflecting & 0.35 & $\sqrt{ }$ \\
HD2r-4 & 1 & 2 & $200 \times 400$ & 1 & 2.58 & 2 & 0.05 & $2 \pi$ & reflecting & 0.46 & $\sqrt{ }$ \\
HD2r-4-HR & 1 & 2 & $400 \times 800$ & 1 & 2.58 & 2 & 0.05 & $2 \pi$ & reflecting & 0.44 & $\sqrt{ }$ \\
HD2r-5 & 1 & 2 & $200 \times 400$ & 1 & 5.16 & 4 & 0.05 & $2 \pi$ & reflecting & 0.52 & $\sqrt{ }$ \\
\hline
\end{tabular}

Notes. The table entries are the same data as Table A.1 with the following exceptions: the column $\boldsymbol{b}_{0}$ is skipped, and we do not list a theoretical value of the growth rate. Instead, we give our choice of boundary conditions in the transverse direction in column "BC". In the last column, we indicate models for which the instability grows oscillatory by a confirmation mark, $\sqrt{ }$. Note that model grw-3 of Table A.1 corresponds to model HD2r-0 with open boundaries.

Table A.3. Initial data and amplification factors of the weak-field models.

\begin{tabular}{|c|c|c|c|c|c|c|c|c|c|c|c|c|c|}
\hline \multirow[t]{2}{*}{ M } & \multirow[t]{2}{*}{$a$} & \multirow{2}{*}{$\begin{array}{r}b_{0}^{x} \\
{\left[10^{-4}\right.}\end{array}$} & \multirow[t]{2}{*}{ A } & \multicolumn{2}{|c|}{256} & \multicolumn{2}{|c|}{512} & \multicolumn{2}{|c|}{1024} & \multicolumn{2}{|c|}{2048} & \multicolumn{2}{|c|}{4096} \\
\hline & & & & $f^{e}$ & $f^{b}$ & $f^{e}$ & $f^{b}$ & $f^{e}$ & $f^{b}$ & $f^{e}$ & $f^{b}$ & $f^{e}$ & $f^{b}$ \\
\hline 0.5 & 0.05 & 200 & 25 & 20.2 & 29.4 & 22.9 & 30.6 & 25.9 & 29.3 & 27.7 & 28.3 & & \\
\hline 0.5 & 0.05 & 100 & 50 & 24.4 & 40.4 & 33.5 & 57.2 & 39.8 & 66.2 & 43.5 & 64.3 & 46.3 & 63.3 \\
\hline 0.5 & 0.05 & 50 & 100 & 27.0 & 50.0 & 41.0 & 75.6 & 55.3 & 102.2 & 66.8 & 123.7 & 73.3 & 125.3 \\
\hline 0.5 & 0.05 & 20 & 250 & 35.0 & 51.0 & 44.4 & 95.0 & 70.4 & 146.4 & 105.3 & 213.0 & & \\
\hline 1 & 0.10 & 200 & 50 & 25.2 & 36.5 & 33.6 & 50.2 & 46.0 & 46.5 & 45.0 & 49.2 & & \\
\hline 1 & 0.10 & 40 & 250 & 18.2 & 37.6 & 49.3 & 83.8 & 74.5 & 132.2 & 113.9 & 201.3 & & \\
\hline 1 & 0.15 & 200 & 50 & 17.2 & 29.3 & 27.8 & 39.7 & 30.7 & 40.0 & 35.9 & 46.4 & & \\
\hline 1 & 0.15 & 100 & 100 & 19.6 & 34.8 & 35.0 & 56.3 & 54.9 & 76.0 & 61.2 & 81.5 & & \\
\hline 1 & 0.15 & 40 & 250 & 21.3 & 46.6 & 40.2 & 69.5 & 65.3 & 106.3 & 103.9 & 152.4 & & \\
\hline 1 & 0.20 & 200 & 50 & 5.8 & 14.2 & 8.0 & 28.0 & 12.8 & 26.0 & 22.5 & 36.3 & & \\
\hline 1 & 0.20 & 40 & 250 & 6.4 & 35.7 & 11.8 & 41.3 & 18.1 & 62.2 & 33.0 & 106.6 & & \\
\hline 1 & 0.05 & 400 & 25 & 16.8 & 23.8 & 19.6 & 25.9 & 22.0 & 26.6 & 23.3 & 25.4 & & \\
\hline 1 & 0.05 & 200 & 50 & 19.4 & 45.7 & 27.5 & 46.2 & 32.0 & 48.6 & 36.1 & 51.5 & 39.4 & 53.6 \\
\hline 1 & 0.05 & 80 & 125 & 20.2 & 35.6 & 33.4 & 70.2 & 50.1 & 96.6 & 61.6 & 117.3 & 67.3 & 118.9 \\
\hline 1 & 0.05 & 40 & 250 & 20.9 & 50.8 & 37.0 & 88.0 & 59.9 & 127.7 & 83.6 & 178.5 & 104.1 & 210.9 \\
\hline 1 & 0.05 & 20 & 500 & 21.2 & 55.1 & 39.4 & 103.1 & 63.0 & 153.1 & 101.1 & 236.4 & 145.6 & 330.8 \\
\hline 1 & 0.05 & 8 & 1250 & 21.2 & 56.4 & 40.1 & 127.2 & 67.7 & 187.8 & 109.8 & 288.7 & 169.0 & 444.4 \\
\hline 1 & 0.05 & 2 & 5000 & 21.2 & 55.2 & 40.3 & 136.4 & 68.6 & 218.5 & 112.4 & 314.7 & 182.9 & 515.2 \\
\hline
\end{tabular}

Notes. The columns give the initial Mach number, M, the shear-layer width, $a$, the initial magnetic field strength, $b_{0}^{x}$, the corresponding Alfvén number, A, and the amplification factors $f^{\mathrm{e}}$ (for the magnetic energy) and $f^{b}$ (for the field strength), respectively. The models were simulated on grids of $m=256, \ldots, 4096$ zones per dimension. 
M. Obergaulinger et al.: Local simulations of the magnetized Kelvin-Helmholtz instabilityin neutron-star mergers

Table A.4. Disruption time and deceleration rate of weak-field models.

\begin{tabular}{|c|c|c|c|c|c|c|c|c|c|c|c|c|c|}
\hline \multirow[t]{2}{*}{ M } & \multirow[t]{2}{*}{$a$} & \multirow{2}{*}{$\begin{array}{r}b_{0}^{x} \\
{\left[10^{-4}\right]}\end{array}$} & \multirow[t]{2}{*}{ A } & \multicolumn{2}{|c|}{256} & \multicolumn{2}{|c|}{512} & \multicolumn{2}{|c|}{1024} & \multicolumn{2}{|c|}{2048} & \multicolumn{2}{|c|}{4096} \\
\hline & & & & $t_{\mathrm{dis}}$ & $\sigma_{\mathrm{dec} ; 3}$ & $t_{\mathrm{dis}}$ & $\sigma_{\mathrm{dec} ; 3}$ & $t_{\mathrm{dis}}$ & $\sigma_{\mathrm{dec} ; 3}$ & $t_{\mathrm{dis}}$ & $\sigma_{\mathrm{dec} ; 3}$ & $t_{\mathrm{dis}}$ & $\sigma_{\mathrm{dec} ; 3}$ \\
\hline 0.5 & 0.05 & 200 & 25 & 7.6 & 19.0 & 7.6 & 23.0 & 7.6 & 18.6 & 7.6 & 22.4 & & \\
\hline 0.5 & 0.05 & 100 & 50 & 14.4 & 10.7 & 13.7 & 11.7 & 12.7 & 14.3 & 12.6 & 11.6 & & \\
\hline 0.5 & 0.05 & 50 & 100 & 80.1 & 4.1 & 45.4 & 5.9 & 23.4 & 7.4 & 22.9 & 10.7 & 22.6 & 10.3 \\
\hline 0.5 & 0.05 & 20 & 250 & - & $\sim 0.4$ & - & $\sim 0.9$ & - & 3.5 & 77.4 & 4.1 & & \\
\hline 1 & 0.15 & 200 & 50 & 4.5 & 19.0 & 4.3 & 16.9 & 4.0 & 16.9 & 4.1 & 24.1 & & \\
\hline 1 & 0.15 & 100 & 100 & 23.0 & 6.8 & 15.0 & 13.4 & 6.5 & 17.1 & 6.7 & 18.1 & & \\
\hline 1 & 0.15 & 40 & 250 & - & $\sim 0.17$ & - & 2.7 & 58.5 & 4.4 & 21.9 & 6.1 & & \\
\hline 1 & 0.05 & 400 & 25 & 3.8 & 23.9 & 3.8 & 22.6 & 3.8 & 45.0 & 3.8 & 41.1 & & \\
\hline 1 & 0.05 & 200 & 50 & 12.4 & 16.8 & 9.9 & 14.1 & 6.1 & 27.8 & 6.0 & 23.0 & & \\
\hline 1 & 0.05 & 80 & 125 & 75.6 & 4.8 & 25.3 & 8.7 & 18.5 & 11.6 & 12.0 & 15.2 & 12.0 & 12.9 \\
\hline 1 & 0.05 & 40 & 250 & - & $\sim 0.9$ & - & 1.8 & 62.5 & 4.1 & 39.8 & 5.6 & 39.8 & 5.6 \\
\hline 1 & 0.05 & 20 & 500 & - & - & - & - & - & $\sim 0.8$ & - & 2.4 & 99.5 & 3.1 \\
\hline 1 & 0.05 & 8 & 1250 & - & - & - & - & - & - & - & $\sim 0.5$ & - & $\sim 0.8$ \\
\hline 1 & 0.05 & 2 & 5000 & - & - & - & - & - & - & - & - & - & - \\
\hline
\end{tabular}

Notes. Same as Table A.3, but instead of the amplification factors we give the disruption time of the KH vortex, $t_{\mathrm{dis}}$, and the absolute value of the deceleration rate, $\sigma_{\mathrm{dec} ; 3}=\left|\sigma_{\mathrm{dec}} / 10^{-3}\right|$, for simulations with $m=256, \ldots, 4096$ zones per dimension. We indicate simulations where no disruption is observed by a hyphen in the column for $t_{\text {dis }}$, simulations where the determination of $\sigma_{\text {dec }}$ is very inaccurate by a sign preceding the value of $\sigma_{\mathrm{dec} ; 3}$, and simulations where we found no measurable deceleration by a hyphen in the column for $\sigma_{\mathrm{dec} ; 3}$.

Table A.5. List of 3D models.

\begin{tabular}{rrrrccc}
\hline \hline$U_{0}$ & $\mathrm{M}$ & $\begin{array}{c}b_{0}^{x} \\
10^{-4}\end{array}$ & $\mathrm{~A}$ & 128 & 256 & 512 \\
& & & & & \\
\hline 1 & 1 & 0 & $\infty$ & + & & \\
1 & 1 & 400 & 25 &,+ \pm &,+ \pm & \\
1 & 1 & 200 & 50 &,+ \pm &,+ \pm & + \\
1 & 1 & 2 & 5000 & + & & \\
1 & 4 & 0 & $\infty$ & + & + & \\
1 & 4 & 400 & 25 & + & + & \\
1 & 4 & 200 & 50 & + & + & \\
1 & 4 & 100 & 100 & + & + & \\
1 & 4 & 20 & 500 & + & + & \\
\hline
\end{tabular}

Notes. The columns give the initial shear velocity, $U_{0}$, Mach number, $\mathrm{M}$, magnetic field strength, $b_{0}$, and Alfvén number $\mathrm{A}$. The models were simulated on grids of $128^{3}$ to $512^{3}$ zones using parallel (+ sign) and anti-parallel ( \pm sign) initial field configurations, respectively. Most of the models were simulated several times using different initial perturbations.

Table A.6. List of 2D merger-motivated models simulated on grids of $1024^{2}$ and $2048^{2}$ zones, respectively.

\begin{tabular}{rccc}
\hline \hline$b_{0}^{x}\left[10^{13} \mathrm{G}\right]$ & \multicolumn{2}{c}{$\mathrm{M}=1$} & $\mathrm{M}=4$ \\
& $1024^{2}$ & $2048^{2}$ & $1024^{2}$ \\
\hline \pm 5 & $\sqrt{ }$ & & \\
10 & $\sqrt{ }$ & $\sqrt{ }$ & \\
\pm 10 & $\sqrt{ }$ & $\sqrt{ }$ & $\sqrt{ }$ \\
20 & $\sqrt{ }$ & & $\sqrt{ }$ \\
\pm 20 & $\sqrt{ }$ & $\sqrt{ }$ & $\sqrt{ }$ \\
40 & & & $\sqrt{ }$ \\
\pm 40 & & & $\sqrt{ }$ \\
\hline
\end{tabular}

Notes. Each simulated model is indicated by a $\sqrt{ }$ sign. The initial shear profile had a maximum velocity of $v_{0}^{x}=1.83 \times 10^{9} \mathrm{~cm} / \mathrm{s}$ (i.e., the Mach number of the shear flow is $M=1$ ), and a width of $a=20 \mathrm{~m}$. The first column lists the initial field strength, marking models with anti-parallel initial fields by a \pm preceding the numerical value. Most models were simulated using different initial perturbations, and most models were additionally simulated on coarser grids.

\section{References}

Agertz, O., Moore, B., Stadel, J., et al. 2007, MNRAS, 380, 963

Anderson, M., Hirschmann, E. W., Lehner, L., et al. 2008, Phys. Rev. Lett., 100, 191101

Baty, H., Keppens, R., \& Comte, P. 2003, Phys. Plas., 10, 4661

Biskamp, D. 2000, Magnetic Reconnection in Plasmas (Cambridge, UK: Cambridge University Press), Cambridge monographs on plasma physics, 3
Table A.7. Same as Table A.6 but for the 3D merger-motivated models simulated on grids of $128^{3}$ and $256^{3}$ zones, respectively.

\begin{tabular}{rcccc}
\hline \hline$b_{0}^{x}\left[10^{13} \mathrm{G}\right]$ & \multicolumn{2}{c}{$\mathrm{M}=1$} & \multicolumn{2}{c}{$\mathrm{M}=4$} \\
& $128^{3}$ & $256^{3}$ & $128^{3}$ & $256^{3}$ \\
\hline 5 & $\sqrt{ }$ & $\sqrt{ }$ & & \\
\pm 5 & $\sqrt{ }$ & $\sqrt{ }$ & & \\
10 & $\sqrt{ }$ & $\sqrt{ }$ & $\sqrt{ }$ & $\sqrt{ }$ \\
\pm 10 & $\sqrt{ }$ & $\sqrt{ }$ & $\sqrt{ }$ & $\sqrt{ }$ \\
20 & $\sqrt{ }$ & $\sqrt{ }$ & $\sqrt{ }$ & $\sqrt{ }$ \\
\pm 20 & $\sqrt{ }$ & $\sqrt{ }$ & $\sqrt{ }$ & $\sqrt{ }$ \\
40 & & $\sqrt{ }$ & $\sqrt{ }$ & $\sqrt{ }$ \\
\pm 40 & & $\sqrt{ }$ & $\sqrt{ }$ & $\sqrt{ }$ \\
\hline
\end{tabular}

Chandrasekhar, S. 1961, Hydrodynamic and hydromagnetic stability, International Series of Monographs on Physics (Oxford: Clarendon)

Evans, C. R., \& Hawley, J. F. 1988, ApJ, 332, 659

Frank, A., Jones, T. W., Ryu, D., et al. 1996, ApJ, 460, 777

Gardiner, T. A., \& Stone, J. M. 2005, J. Comput. Phys., 205, 509

Gardiner, T. A., \& Stone, J. M. 2008, J. Comput. Phys., 227, 4123

Giacomazzo, B., Rezzolla, L., \& Baiotti, L. 2009, MNRAS, 399, L164

Harten, A. 1983, J. Comput. Phys., 49, 357

Iroshnikov, P. S. 1964, SvA, 7, 566

Jeong, H., Ryu, D., Jones, T. W., et al. 2000, ApJ, 529, 536

Jones, T. W., Gaalaas, J. B., Ryu, D., et al. 1997, ApJ, 482, 230

Keil, W., Janka, H.-T., \& Müller, E. 1996, ApJ, 473, L111

Keppens, R., Tóth, G., Westermann, R. H. J., et al. 1999, J. Plasma Phys., 61, 1

Kraichnan, R. H. 1965, Phys. Fluids, 8, 1385

LeVeque, R. J. 1992, Numerical Methods for Conservation Laws, 2nd edn., Lectures in mathematics - ETH Zürich (Birkhäuser)

Levy, D., Puppo, G., \& Russo, G. 2002, SIAM J. Sci. Comput., 24, 480

Liu, X.-D., Osher, S., \& Chan, T. 1994, J. Comput. Phys., 115, 200

Liu, Y. T., Shapiro, S. L., Etienne, Z. B., et al. 2008, Phys. Rev. D, 78, 024012

Londrillo, P., \& del Zanna, L. 2004, J. Comput. Phys., 195, 17

Miura, A., \& Pritchett, P. L. 1982, J. Geophys. Res., 87, 7431

Monaghan, J. J. 1992, ARA\&A, 30, 543

Obergaulinger, M. 2008, Ph.D. Thesis, Technische Universität München

Obergaulinger, M., Cerdá-Durán, P., Müller, E., et al. 2009, A\&A, 498, 241

Oechslin, R., Janka, H.-T., \& Marek, A. 2007, A\&A, 467, 395

Orszag, S. A., \& Tang, C.-M. 1979, J. Fluid Mech., 90, 129

Price, D. J., \& Rosswog, S. 2006, Science, 312, 719

Rosswog, S. 2007, in Rev. Mex. Astron. Astrofis. Conf. Ser., 27, 57

Ryu, D., \& Jones, T. W. 1995, ApJ, 442, 228

Ryu, D., Jones, T. W., \& Frank, A. 2000, ApJ, 545, 475

Suresh, A., \& Huynh, H. 1997, J. Comput. Phys., 136, 83

Titarev, V. A., \& Toro, E. F. 2005, Int. J. Numer. Meth. Fluids, 49, 117

Toro, E. F., \& Titarev, V. A. 2006, J. Comput. Phys., 216, 403 